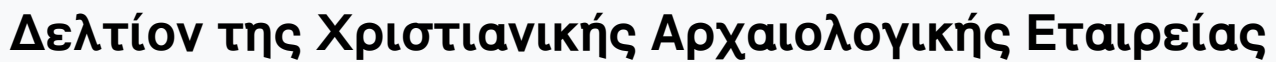

Tó 13 (1988)

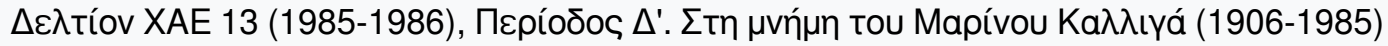

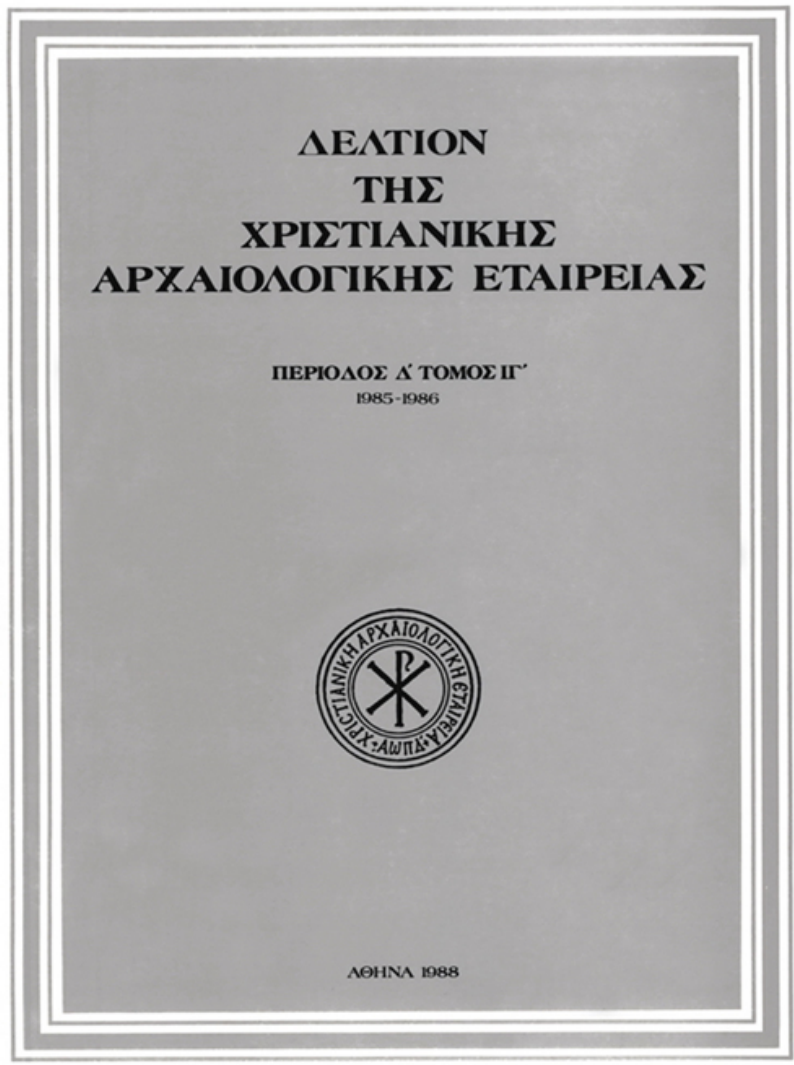

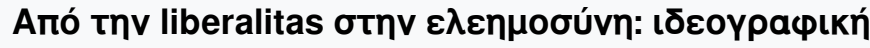

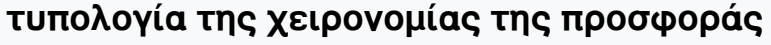

Élias ANTONOPOULOS

doi: $10.12681 /$ dchae.995

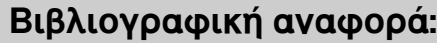

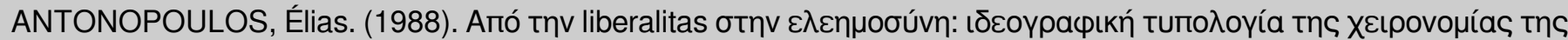

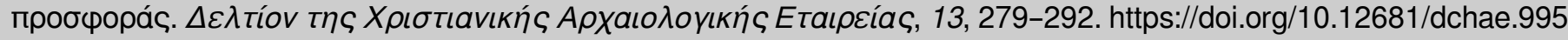




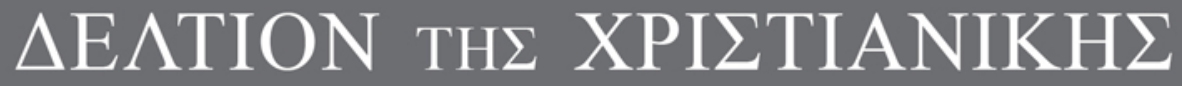 APXAIO $\Lambda$ OГIKH $\Sigma$ ETAIPEIA $\Sigma$}

De la Libéralité à l'Aumône: Typologie idéographique du geste donateur

Élias ANTONOPOULOS

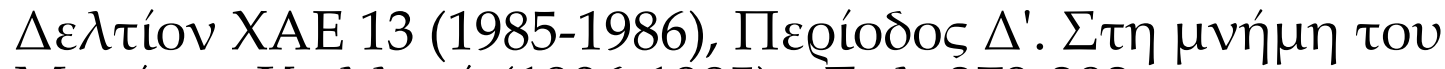

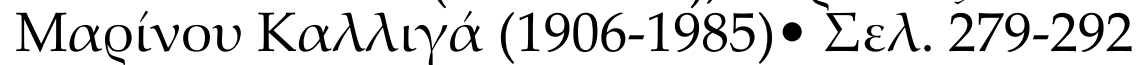

A@HNA 1988 


\section{DE LA LIBÉRALITÉ A L'AUMÔNE: TYPOLOGIE IDÉOGRAPHIQUE DU GESTE DONATEUR}

$\mathrm{D}$ epuis l'essai fondamental, sur le don, de Marcel Mauss, plus près de nous, les ouvrages de Paul Veyne et d'Evelyne Patlagean, concernant respectivement l'évergétisme antique et la pauvreté à Byzance, les travaux de Michel Mollat sur les pauvres, pour ce qui concerne l'Occident médiéval, en passant par l'étude de Demetrios Constantelos sur la philanthropia byzantine, depuis la parution de ces travaux, et de plusieurs autres, s'est construite une thématique historique amplement travaillée, qui devrait appuyer des enquêtes visant des objectifs correspondants, recherchés dans le domaine des images ${ }^{1}$.

Dans ce cadre de projet de construction d'un inventaire, seront ici regroupées des images répondant à un critère iconographique, qui correspond à un geste (et là, nous avons encore un répertoire à dresser): le geste manuel de répandre (spargere) de l'argent, geste activé par un personnage important en faveur d'une foule de bénéficiaires. La fréquence chronologique des images rassemblées est faible, leur instrumentalité dans la construction du "système" de l'iconographie byzantine n'est pas apparente, et d'ailleurs, elles ne captivent pas de première vue l'attention du chercheur sérialiste; mais elles attendent à être enregistrées dans toute la diversité, à la fois de leurs manifestations et de leurs témoignages ${ }^{2}$.

Notre enquête, dans sa volonté de saisir certaines motivations, ou de visualiser le milieu de rayonnement de la largesse pécuniaire, sera appuyée sur le témoignage du Satiricon. La conduite en question est mise en oeuvre par le geste large (largitio); dirigée par un individu, elle vise un certain nombre de "clients" de sa zone d'activité, dans un climat de vanité grandiloquente ${ }^{3}$. C'est l'intéressé lui-même qui dresse ici le répertoire de ses libéralités; on est déjà très loin des actes fondateurs de l'évergétisme antique ${ }^{4}$. Lors d'un festin donné dans une villa en Italie méridionale, aux environs de Naples, Trimalcion s'adresse à son collègue, le sévir Habinnas — qui est aussi marbrier: lapidarius -, pour commander l'iconographie de son monument funéraire, qui doit exalter le rang du supposé défunt, les richesses qu'il a accumulées et les munificences dont il a fait preuve envers ses concitoyens ${ }^{5}$. Parmi les motifs les plus évocateurs de sa vie, il s'imagine lui-même "siégeant sur un tribunal, et distribuant au peuple un sac d'écus"6. L'on pourrait illustrer cette description par des monuments qui vont du milieu du premier' siècle avant au milieu du premier siècle après Jésus-Christ, et qui illustrent à leur tour ce courant d'art que Bianchi Bandinelli nomma plébéien?; plus tard, sous la Tétrarchie, ce même courant modèlera l'art officiel $^{8}$.

Par ce répertoire sépulcral, Trimalcion espère réserver sa place dans la mémoire de ses concitoyens. Le geste généreux, banalisé par le personnage de Pétrone, est calqué sur le modèle donné par le prince. C'est par le prince également, lors de sa transformation en dominus, que sera sublimé le même répertoire dans l'art officiel ${ }^{9}$. Mais auparavant, pour obtenir à leur point de départ les éléments qui composeront cette thématique iconographique, on remontera vers ces images de l'art romain

1. M. Mauss, Essai sur le don. Forme et raison de l'échange dans les sociétés archaïques, Année Sociologique, seconde série, 1 (19231924); P. Veyne, Le pain et le cirque. Sociologie historique d'un pluralisme politique, Paris 1976; E. Patlagean, Pauvreté économique et pauvreté sociale à Byzance, IVe-VIIe siècles, Paris-La Haye 1977; M. Mollat, Les pauvres au moyen âge. Etude sociale, Paris 1978; cf. id. (éd.), Etudes sur l'Histoire de la pauvreté, Paris 1974 (2 vol.); D. Constantelos, Byzantine Philanthropy and Social Welfare, New Brunswick, N.J., 1968. Certaines des pièces les plus importantes du dossier de la libéralité ont été commentées dans une communication: The 17th International Byzantine Congress (Washington, D.C., 1986), Abstracts of Short Papers, p. 13. D'autres documents, qui s'y rattachent directement ou indirectement, seront séparément présentés. 2. Cf. E. Antonopoulos, Miséricorde, olivier: agents et attributs, Byzantion 51 (1981), p. 345-385).

3. Satiricon 71; cf. Pét rone, Le Satiricon, éd. A. Ernout, Paris $1950^{3}$. Pour l'identité sociale de l'assistance, cf. P. Veyne, Vie de Trimalcion, Annales 16 (1961), p. 241 (non pas des clients, mais des esclaves et des amis).

4. Cf. à ce sujet Veyne 1976 (n. 1, supra).

5. Satiricon 71, 5 sq. Cf. A. Maiuri, La cena di Trimalchione di Petronio Arbitro, Naples 1945. Au sujet du sévir Habinnas qui exerce le mêtier de marbrier, cf. Satiricon 65, 5. Pour le testament de Trimalcion, que celui-ci n'a pas manqué de lire "d'un bout à l'autre ....au milieu des gémissements de toute la maison" (Satiricon 71, 4), ainsi que pour le monument sépulcral qu'il commanda à Habinnas, cf. Maiuri, ibid., p. 79-81, 209-212.

6. Satiricon 71, 9-10: et me in tribunali sedentem praetextatum cum anulis aureis quinque et nummos in publico de sacculo effundentem (éd. Ernout, p. 72). Cf. Maiuri, ibid., p. 211-212; Veyne 1961 (n. 3, supra), p. 242.

7. R. Bianchi Bandinelli, Dall'ellenismo al medioevo, Rome, 1978 , p. $40-41$; fig. 11,14

8. Cf. ibid., p. 64-65.

9. Cf. n. 11, infra. 
qui enregistrent les distributions gracieuses ${ }^{10}$. Le schéma des congiaria, connu par les monnaies, est reproduit dans le domaine de l'art monumental, avec l'un des reliefs marc-auréliens, ultérieurement encastrés sur l'arc de Constantin, à Rome. On y voit, présenté de trois quarts, le déroulement iconique de la Liberalitas Augus$t i$, qui se développe en deux étapes, de l'opérateur au bénéficiaire, en passant par un intermédiaire (Fig. 1). C'est sur le même monument que figure la Libéralité constantinienne; l'empereur est ici vu de front, au milieu d'une composition symétrique ${ }^{11}$.

Enfin, le développement en deux phases de l'image plus ancienne se trouve comprimé dans une formule à personnage unique, seul ou dans un contexte subsidiaire, avec l'image de Constance II sur la célèbre page du Calendrier de 354 (Fig. 2); il faut cependant préciser qu'il s'agit maintenant d'un événement spécial (la sparsio consulaire), et que cet événement est dans ce cas figuré dans un codex ${ }^{12}$. Par cette condensation du sensible est dilaté le potentiel intelligible, et c'est ainsi que nous abordons le domaine des images symboliques. Constance II - qui assuma la charge de consul en 354figure nimbé, de face, trônant, répandant des monnaies, inscrit à l'intérieur d'un édicule qui encadre sa magnificence. Ce déclenchement de la pluie d'or se répercute surtout dans des images monétaires du IVe siècle, où l'on peut voir l'empereur en aurige, debout, vu de front sur son char, venant directement à la rencontre du spectateur, et irradiant par les monnaies qu'il lance ${ }^{13}$. Le même type iconographique est reproduit plus tard (VIe s.), sur le médaillon d'or de la Collection de Dumbarton Oaks (Fig. 3), où l'on peut reconnaître le geste aspergeant de monnaies ${ }^{14}$. C'est cette version surtout du geste donateur que vise notre approche actuelle.

La transition, dans les images, d'un personnage historique, vu de face, dans la mise en scène de la sparsio (Fig. 2 ), vers l'abstraction personnifiée de sa vertu, figurée seule ou conjointement avec lui, est opérée dans deux ouvres paléobyzantines (Fig. 4, 5). Les exemples précédemment cités marquent certaines étapes de l'itinéraire menant du concret actuel (libéralité documentaire) à l'abstrait qui prête à réflexion (libéralité symbolique).

Au milieu du Ve siècle, le geste en question est réactivé par une figure féminine vue en buste, frontalement, dans un médaillon, épicentre à la fois plastique et sémantique d'une mosaïque de pavement à Yaktô, l'ancienne Daphnè -faubourg d'Antioche (Fig. 4). Elle répand, avec sa droite, des monnaies, puisées dans un récipient, la sporta, qu'elle tient de sa gauche; or, l'exiguïté de l'espace contracte le développement du geste: les monnaies restent dans le creux de la main ${ }^{15}$. Autour d'elle, dans un champ quadrangulaire, sont disposées des scènes de combats de venatores favoris du moment
10. D. va n Berchem, Les distributions de blé et d'argent à la plèbe romaine sous l'Empire, Genève 1939, p. 119 sq. Pour la différence entre largitio et congiaire, cf. H. Stern, Le Calendrier de 354. Etude sur son texte et ses illustrations, Paris 1953, p. 163. Au sujet des largesses impériales, cf. R. MacMullen, The Emperor's Largesses, Latomus 21 (1962), p. 159-166.

11. Deux Libéralités de date différente ont été regroupées sur l'arc de Constantin, construit après la victoire contre Licinius: d'une part, la Libéralité de Marc-Aurèle, figurée sur l'un des reliefs qui proviennent d'un monument antérieur; cette composition commémore peut-être un événement de l'année 173. Cf. G. Becat ti, Il rilievo della Liberalitas di Marco Aurelio, Archeologia Classica 24 (1972), p. 59-74; cf. également id., La colonna coclide istoriata, Rome 1960, p. 291 sq., pl. 11; R. Brilliant, Gesture and Rank in Roman Art. The Use of Gestures to Denote Status in Roman Sculpture and Coinage, New Haven, Connecticut, 1963, p. 152, fig. 3.117; Bianchi Bandinelli 1978 (n. 7, supra), p. 107-108, fig. 62. D'autre part, sur le même monument figure la Libéralité de Constantin; voir H. P. L'Orange - A. von Gerkan, Der spätantike Bildschmuck des Konstantinsbogens, Berlin 1939, p. 89-102; Brilliant, ibid., p. 172, fig. 4.21; Bianchi Bandinelli, ibid., p. 65, 105, fig. 59; id., Roma. La fine dell'arte antica, Milan $1976^{2}$, p. 76-77, 79-80, fig. 70 (vues partielles). En dernier lieu, cf. New York, The Metropolitan Museum of Art, 19 nov. 1977-12 févr. 1978, Age of Spirituality. Late Antique and Early Christian Art, Third to Seventh Century, Edited by K. Weitzmann, 1979, n 58 (Brilliant). Pour la générosité de Constantin, cf. G. Dagron, Naissance d'une capitale. Constantinople et ses institutions de 330 à 451, Paris 1974, p. 34-35.

12. Stern 1953 (n. 10, supra), p. 152 sq. Pour la sparsio consulaire, cf. ibid., p. 155 sq.

13. Cf. les exemples de Fest-aurei reproduits dans J. Toynbee, Roman Medallions, New York 1944, pl. II.15-16 (Constantin), II.17 (Constance II), III.1 (Constant), III.2 (Valentinien I), III.3 (Valentinien II); cf. Stern, ibid., p. 156, pl. 31.10-11 (Constance II, Constant).

14. Le relief de cette plaque est obtenu au repoussage, et il est modelé sur une médaille, peut-être de Maurice-Tibère, pour servir au montage d'un bracelet. Voir M. Ross, Catalogue of the Byzantine and Early Mediaeval Antiquities in the Dumbarton Oaks Collection, vol. 2, 1965, no 2A (p. 4-6, pl. 4); cf. id., A Byzantine Gold Medallion at Dumbarton Oaks, DOP 11 (1957), p. 256, fig. 8; A. Cameron, Porphyrios the Charioteer, Oxford 1973, p. 22, 25, pl. 31.12. Pour le nouvel an dans l'Empire chrétien, cf. M. Meslin, La fête des kalendes de janvier dans l'Empire romain. Etude d'un rituel du nouvel an, Bruxelles 1970, p. 51 sq.; sur la sparsio consulaire, opérée le ler janvier, cf. ibid., p. 59-61, 69, 102. Toujours sur les sparsiones, cf. M. Hendy, Studies in the Byzantine Monetary Economy c. 300-1450, Cambridge 1985, p. 193 sq.

15. Il ne s'agit bien entendu que d'une convention imposée par le cadre. Il revient à Doro Levi d'avoir été le premier à reconnaître que le geste de la figure du médaillon est modelé sur la sparsio consulaire: D. Levi, Antioch Mosaic Pavements, Princeton 1947, vol. 1, p. 339; par ailleurs, le même auteur reporte le récipient tenu par Mégalopsychia aux sportae dont parle Corippus, Iust. 4, 105 (ibid., p. 340); cf. maintenant l'édition d'Averil Cameron (Flavius Cresconius Corippus, In laudem Iustini Augusti minoris, Libri IV, Londres 1976). Au sujet du même contenant cf. Becatti 1960 (n. 11, supra), p. 295-296. La novelle 105 de Justinien retient le diminutif du même terme (sportula): Quantacumque ergo competens est dari ab eo qui a nobis eligitur per annum consule occasione sportularum ( $\pi \rho \circ \varphi \alpha \dot{\sigma \varepsilon \iota}$ $\sigma \pi \circ \rho \tau o u ́ \lambda \omega v$ ) omnium et distributionum et expensarum ... (Nov. 105, 1; éd. Schoell - Kroll, p. 501.32-34). Cf. les contenants figurés sur les médailles impériales: Levi, ibid., p. 340, fig. 138; le même auteur nous 


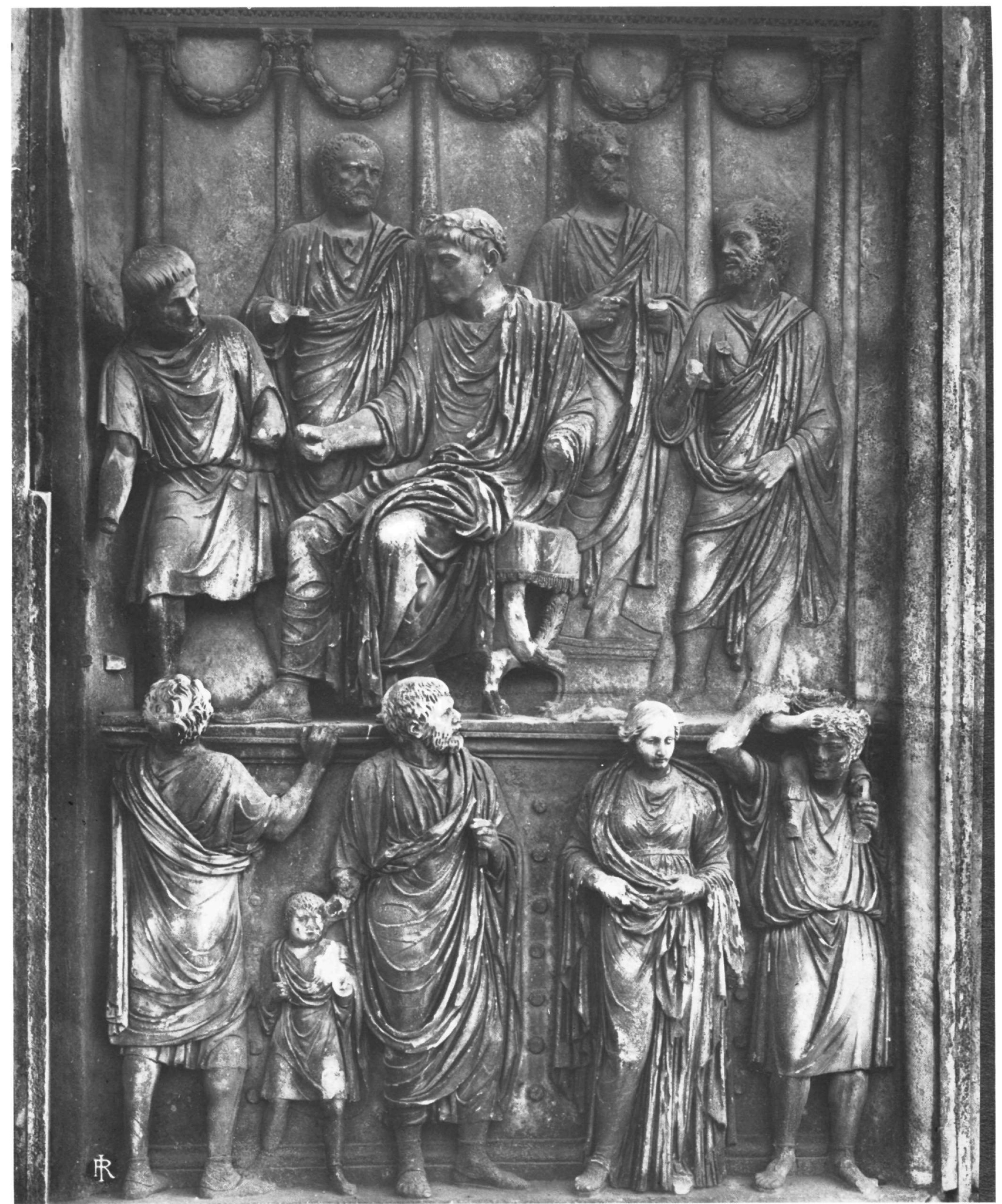

Fig. 1. Rome, Arc de Constantin. Panneau sculpté de la Libéralité de Marc-Aurèle 


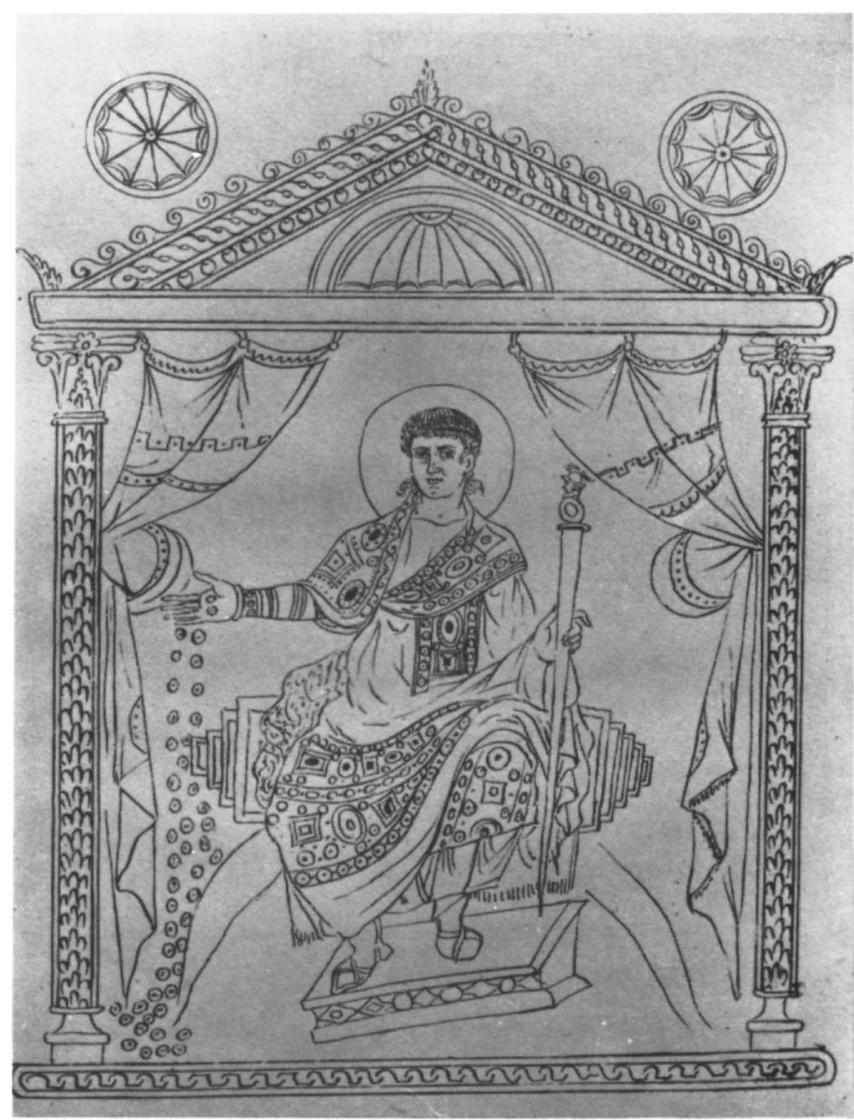

Fig. 2. Rome, Bibl. Vaticane. Cod. Barber. lat. 2154 (Calendrier de 354: copie de Peiresc). Fol. 13: Constance II répandant des monnaies

- qui portent des pseudonymes mythologiques - contre des fauves, tandis que l'ensemble est bordé par une frise où se déroulent des scènes de vie urbaine, et où l'on peut reconnaître certains monuments de la cité ${ }^{16}$. La réapparition du geste du côté hellénophone de l'Empire est assortie d'une nouvelle identité, qui renvoie à une vertu aristotélicienne; la figure du médaillon est nommée Mégalopsychia: Magnanimité ${ }^{17}$. Les deux composantes de ce terme, sont inscrites, séparées l'une de l'autre, de part et d'autre de sa tête (MEГA $\Lambda$ O - $\Psi$ YXIA). Présentant la magnanimité en personne pour la première fois, au moins dans les monuments préservés, la mosaïque de Yaktô est le produit d'une période —et d'une aire culturelle - où les personnifications d'abstractions ont singulièrement proliféré ${ }^{18}$.

Prolongeant la valeur opérationnelle de la vertu jusque dans l'espace figuré qui entoure son portrait fictif, on pourrait être enclin à supposer - face aux combats figurés- qu'il s'agit d'exemples de "magnanimité" héroïque qui dépeignent la phase "fondatrice" de la lutte de l'homme contre la bête, de la vertu contre le vice, en plusieurs séquences regroupées simultanément à l'inté- rieur du même cadre, et chaque fois réactivées par des bestiaires combattant contre des fauves, deux par deux. Le point culminant de l'instauration d'un ordre pacifique, présidé "personnellement" par Mégalopsychia dans sa manifestation de générosité, serait imagé au centre du champ figuré. Cette lecture, reconnaissant une valeur allégorique dans les combats contre les bêtes féroces, est certes fondée sur une vaste érudition ${ }^{19}$. On peut cependant objecter que ces combats renvoyaient tout simplement à l'actualité des spectacles donnés en cité $^{20}$.

Il est sans doute certain que pour la mentalité chrétienne les phénomènes perdent leur sens quand on n'y discerne pas une signification implicite - condition préalable de leur formation. Dans cette optique, les combats en question ne sont concevables que s'ils figurent la lutte contre les passions. Quand les mécanismes qui produisent ce raisonnement sont établis dans les consciences, la simultanéité des deux plans est automatique.

Ce ne pouvait pas être pourtant le cas avec un public qui baignait toujours dans les habitudes de la cité "pré-chrétienne".

Du terme personnifié jaillit pour l'historien tout un faisceau de significations, dont une partie seulement -incarnant la dominante contemporaine des mosaïques et/ou s'exprimant comme le désirait le commanditaire- est cristallisée dans l'identité du personnage fi-

rapelle d'ailleurs que la sparsio n'est pas directement vue sur les diptyques consulaires, mais qu'elle y est insinuée par des contenants d'argent (ibid., p. 339-340). Cf. les exemples que nous procurent les illustrations de la Notitia dignitatum: R. Delbrueck, Die Consulardiptychen und verwandte Denkmäler, Berlin - Leipzig 1929, p. 4, fig. 1c (cité par Levi, ibid., p. 109); ibid., Planches, passim, pour le même récipient sur les diptyques consulaires. Sur la mosaïque de Yaktô, l'objet usuel devient attribut, évoquant une source d'abondance. Sur les récipients en forme de sacs, ainsi que sur les libéralités impériales, cf. G. Dagron - C. Morrisson, Le kentènarion dans les sources byzantines, RN 17 (1975), passim. Sur les illustrations de la Notitia dignitatum cf. P. Berger, The Insignia of the Notitia Dignitatum, New York - Londres 1981.

16. G. Downey, A History of Antioch in Syria, from Seleucus to the Arab Conquest, Princeton 1961, p. 30-32, 659-664; cf. J. Lassus, Antioche en 459, d'après la mosaïque de Yakto, Apamée de Syrie. Bilan des recherches archéologiques 1965-1968 (Actes du Colloque tenu à Bruxelles les 29 et 30 Avril 1969), Bruxelles 1969, p. 140. Pour l'ensemble de la mosaïque, cf. Levi, ibid., fig. 136.

17. Cette transition est donc accomplie sur des images produites dans l'aire culturelle hellénique; la vertu mise en valeur, opérant la sparsio (Fig. 4), ou révélant l'arrière plan psychologique d'une personne historique (voir plus loin: Fig. 5), s'y trouve identifiée avec la mégalopsychia aristotélicienne; cf. Aristotelis Ethica Nikomachea, éd. Susemihl,

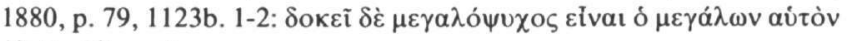

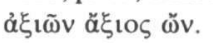

18. Pour les abstractions personnifiées cf. surtout les travaux de 
Glanville Downey (Personifications of Abstract Ideas in the Antioch Mosaics, TransactAmPhilAss 69 (1938), p. 349-363; Representations of Abstract Ideas in the Antioch Mosaics, Journal of the Hist. of Ideas 1 (1940), p. 112-113; Ethical Themes in the Antioch Mosaics, Church History 10 (1941), p. 367-376; cf. id. 1961-n. 16, supra-, p. 33-34) et de Doro Levi (-n. 15, supra-, passim). Plus particulièrement pour la Mégalopsychia de Yaktô et son contexte matériel et historique, voir J. Lassus, La mosaïque de Yaktô, Antioch-on-theOrontes, I, Princeton - Londres - La Haye 1934, p. 114-156; G. Downey, The Pagan Virtue of Megalopsychia in Byzantine Syria, TransactAmPhilAss 76 (1945), p. 279-286; et surtout Levi, ibid., p. 337 sq. Cf. n. 20, infra.

19. Je pense particulièrement à la documentation solidement articulée que nous procure Doro Levi dans sa magistrale étude des mosaïques de pavement d'Antioche (n. 15, supra). Sans rattacher la vertu figurée à l'arsenal moral d'une personne historique, ni à un événement, situé en l'espace de l'amphithéâtre, qui serait produit par la volonté d'une personne hypothétique, Levi emprunte à mégalopsychia sa signification initiale (aucunement attestée par l'image du médaillon), pour l'infuser aux groupes en lutte qui occupent l'espace figuré - afin de les interpréter, par ce moyen, comme des allégories de la magnanimité héroïque. En même temps, cet auteur fut le premier à reconnaître l'évidente version de libéralité magnanime que nous projette directement l'image du médaillon (ibid., p. 339), pour laquelle d'ailleurs, il nous procure des documents théoriques (cf. $\mathrm{n}$. 21 , infra, au sujet des valeurs sémantiques), qui en précisent l'identité. Tout était mis en place pour reporter la composition aux spectacles qu'avec plus ou moins de fréquence l'on pouvait espérer voir en cité; rappelons que c'était déjà l'avis d'E. Weigand, en 1935 (BZ 35, p. 428). Or, Levi opta pour l'interprétation allégorique. Cf. Veyne 1976 (n. 1, supra), p. 339-340 n. 129.

20. Cf. les interprétations de Lassus 1934 (n. 18, supra); id. 1969 (n. 16, supra). En 1969, Lassus proposait de dater cette mosaïque d'immédiatement avant ou après le tremblement de terre de 458 ; le terminus post quem non serait l'année 466, date de l'élimination d'Ardabour, dont les thermes privées figurent sur la frise du périmètre: $\tau$ ò

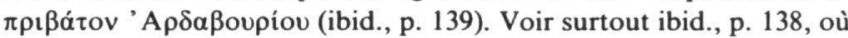
Lassus, malgré son interprétation initiale (1934), propose de voir sur le médaillon central Mégalopsychia présidant aux venationes, il la rapporte par conséquent au donateur des jeux, dont elle serait la vertu principale (ibid., p. 139-140); autrement, la figure allégorique pourrait évoquer les fonds qui ont été dépensés lors de la reconstruction de la ville, après le séisme de 458 (ibid.). Faute de mieux, ni l'une ni l'autre hypothèse ne sont à exclure. Si l'on reportait Mégalopsychia à la reconstruction de la ville, l'on obtiendrait un précédent parallèle de la philoktistos Juliana Anicia (Fig. 5); si on la rattachait à la munificence du donateur de jeux, l'on obtiendrait également un précédent parallèle, mais cette fois de l'époux de Juliana Anicia, le consul Flavius Areobindus Dagalaifus (cf. infra, n. 29). Au sujet de la chasse allégorique, cf. G. Downey, The Pilgrim's Progress of the Byzantine Emperor, Church History 9 (1940), p. 207-217; A. Grabar, L'empereur dans l'art byzantin, Strasbourg 1936, p. 58 sq.; ibid., p. 133 sq.; Levi, ibid., p. 243, 340 sq., 344-345. Cf. Stern 1953 (n. 10, supra), p. 157, qui mentionne la mosaïque de Yaktô dans sa discussion de la sparsio; cet auteur reporte, à titre d'hypothèse, l'image en question à la sparsio consulaire d'Ardabour, en 434 (pour le même Ardabour, cf. ibid., p. 140-144). Cf. I. Lavin, The Hunting Mosaics of Antioch and their Sources. A Study of Compositional Principles in the Development of Early Mediaeval Style, DOP 17 (1963), p. 278, n. 441, qui nous fait remarquer les accents allégoriques de la composition de Yaktô, qui accusent un contraste avec les œuvres de l'Occident, conçues dans un esprit de narration réaliste. Cf. aussi Patlagean 1977 (n. 1, supra), p. 183 (magnificence du donateur), 187-188 (répertoire urbain). En der-

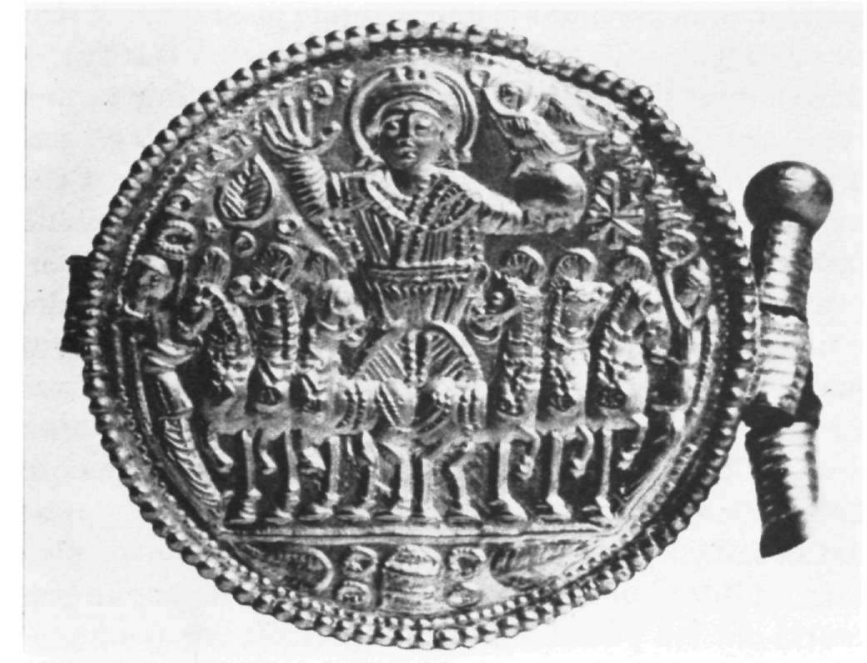

Fig. 3. Washington, Dumbarton Oaks. Médaillon en or repoussé. VIe s. Empereur victorieux, sur char, répandant des monnaies

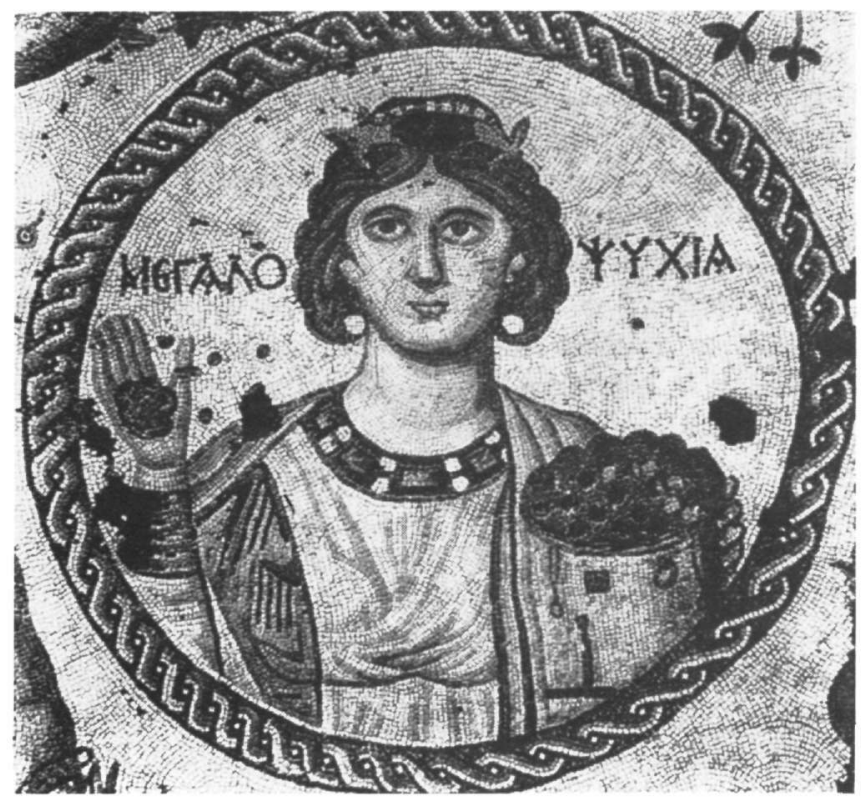

Fig. 4. Antioche, Hatay müzesi. Mosaïque de Yaktô (Daphnè). M. Ve s. Médaillon de la Mégalopsychia

nier lieu, voir K. Dunbabin, The Mosaics of Roman North Africa Studies in Iconography and Patronage, Oxford 1978, p. 228, qui rappelle certaines analogies, reconnues également par Lassus (1969, p. 139), qui existent entre la mosaïque de Mégalopsychia et la mosaïque de Magérius, provenant de Smirat (ibid., p. 67-69, fig. 53), de deux siècles antérieure, et exécutée dans un esprit de "réalisme narratif" (cf. Lavin, cité ci-dessus), contrastant avec le "symbolisme" de la mosaïque de Yaktô. Cf. J. Aymard, La Mégalopsychia de Yaktô et la Magnanimitas de Marc-Aurèle, REA 55 (1953), p. 301-306; id., Les chasses romaines. Des origines à la fin du siècle des Antonins, Paris 1961, p. 191, 302, 405, 439. Sur Ardabour, cf. J. R. Martindale, The Prosopography of the Later Roman Empire, II: A.D. 395-527, Cambridge 1980 , p. 135-137. 
guré; celui-ci personnifie une moralité que l'on peut sentir déduite de tous les points périphériques vers le centre de la composition, sa raison sémantique, définie en une seule figure qui idéalise le concept ${ }^{21}$. Il ne s'agit pas d'une entité dynamique qui déclencherait autour d'elle une agitation périphérique; mais il s'agit d'une idéalité immanente - picturale - , "clef de voûte" d'une mappemonde civile, sans références qui la feraient dépendre d'un au-delà; cet au-delà pourtant, évoqué notamment par la deuxième composante du terme, ne manque pas de résonner dans l'image. Une lecture causale mènerait ainsi de la périphérie vers le centre. Les éléments qui ont habité le champ de la mosaïque (venationes ${ }^{22}$, faune discordante - bête contre bête- et animaux de remplissage, et flore qui sert surtout à départir l'espace en secteurs) ont été puisés dans des répertoires de plein emploi: décor fait d'éléments disponibles, illustrations zoologiques pertinentes, spectacles de combats comme d'habitude pour les contemporains ${ }^{23}$, le tout bordé par un périmètre - celui-ci d'une originalité patente - où se déroule un itinéraire à travers les mirabilia d'une micropolis, qui elle-même s'inscrit dans la macropolis syrienne ${ }^{24}$. Mégalopsychia apparaissant au centre de l'ensemble, ayant emprunté des gestes et des attributs consulaires, traduit en image la vertu principale du propriétaire de la villa où se trouve ce pavement, sa générosité manifestée envers ses concitoyens en leur donnant des spectacles ${ }^{25}$. La main prometteuse du personnage allégorique réactive ad infinitum le geste par lequel se manifeste la grandeur d'âme du personnage loué ${ }^{26}$. Il s'agit probablement d'Ardabour, grand dignitaire militaire de l'Empire — qui, cependant, est absent de la composition ${ }^{27}$.

Se rapportant "latéralement" à la mosaïque de Yaktô, l'image de dédicace sur le fol. $6 \mathrm{v}$ du Dioscoride de Vienne, codex prestigieux datant du début du VIe siècle, réserve une place importante à Mégalopsychia, toujours dans son acception de libéralité 28 .

Or, la figure allégorique se retire maintenant à gauche, et recule simultanément dans l'arrière plan de la démonstration picturale, qui est en revanche présidée par un personnage historique, dont la main droite opère directement la largesse donatrice, mais en la présence de "souffleurs" fictifs (Fig. 5).

Plus précisément, Mégalopsychia y apparaît en acolyte d'une princesse, Juliana Anicia, petite-fille de Valentinien III et épouse du consul d'Orient Flavius Areobindus Dagalaifus ${ }^{29}$; assise (?) à sa droite, sans perdre par conséquent sa prédominance, au moins implicitement, elle rend personnifié le trait le plus prononcé de son caractère. D'autre part, Juliana Anicia, siégeant au milieu, sur la sella curulis consulaire, est accompagnée, sur sa gauche, par Phronèsis (ФPO-
NHCIC: Prudence), qui a pour fonction de tempérer la largesse et d'équilibrer visuellement la composition ${ }^{30}$. En dehors de la Magnanimité et de la Prudence qui développent en éventail la majesté de la personne centrée, dans la composition du codex de Vienne sont encore regroupés autour d'elle deux autres figurants fictifs; ce double surcroît symbolique entre en jeu pour se rattacher spécifiquement à la libéralité de la princesse. Se situant, l'un à côté de l'autre, à sa droite (Fig. 5), lieu de l'opération, ainsi que, sur un second plan, lieu d'apparition de Mégalopsychia, ces deux personnages, figurés en échelle réduite, précisent par leur participation l'identité des bénéficiaires, les arts, dont d'ailleurs un enregistrement par métier s'inscrit en huit séquences, reléguées à la périphérie et peintes en monochromie, sur les secteurs qui encerclent le champ pictural proprement dit. En rapport direct avec la main donatrice, le putto qui se dresse, vu de côté, devant Mégalopsychia - celleci l'air absent-, recueille dans un codex les monnaies qui proviennent du sein de cette dernière ${ }^{31}$; l'inscription qui le surmonte nous apprend qu'il s'agit du "Désir de

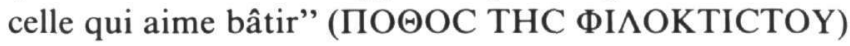
- de la donatrice (philoktistos), s'entend. D'autre part, devant Pothos (par rapport au spectateur), une femme vêtue de blanc et voilée se jette aux pieds de la princesse pour lui rendre hommage au nom des arts, dont elle personnifie la reconnaissance (EYXAPICTIA TE$\mathrm{XN} \Omega \mathrm{N}$ ). Elle est le seul participant qui ne figure pas un trait de caractère du personnage historique; mais venant de dehors, pour s'intégrer dans le même champ pictural, elle personnifie l'écho reconnaissant des arts ${ }^{32}$.

Dans la juxtaposition nuancée des trois figures principales, on peut reconnaître un mélange organique, décomposé dans l'image en ses ingrédients constituteurs, par le moyen des deux figures latérales - qu'englobe au mitan la personne médiane.

Il devient donc évident qu'il s'agit d'une analyse picturale de l'idiosyncrasie du personnage historique. En effet, Mégalopsychia ne totalise pas la vertu de Juliana Anicia, mais elle se partage son arrière plan psychologique avec Phronèsis: face à la polysémie de la personne humaine, nous avons ainsi l'univocité de la personnification. Plus spécifiquement, la magnanimité de la princesse est rattachée expressis verbis à ses activités de bâtisseur d'église; s'intégrant dans l'ensemble, une incription témoigne à cet effet, mentionnant la fondation d'une église au faubourg Honoratae de Constantinople ${ }^{33}$. Rapellons à ce sujet que l'empereur, en tant que bâtisseur ou rénovateur permanent ${ }^{34}$, organise et décore l'espace de la cité; nous sommes toujours, bien entendu, dans la haute époque, qui n'est pas encore coupée de l'antiquité. Par contre, cet ordre d'activités est réduit pendant la période moyenne, où 
21. Voir l'ample discussion de l'évolution sémantique par Doro Levi (n. 15, supra), p. 340 (définition aristotélicienne), 341 (introversion de la vaillance), 342-343 (applications romaines; a) magnanimité active: fortitudo; b) magnanimité passive: patientia; auxquels on ajoutera c) clementia, qui tempère l'exploit martial du victorieux; pour finir avec la liberalitas in usu pecuniae). Si l'on percevait dans l'étude de Levi un manque, ce serait peut-être au sujet de l'arrière plan discursif, contemporain à cette image, à Antioche même; cet auteur repère toutefois les jointures de l'expérience qui informa la pensée romaine, à partir des données qu'avait façonné le monde hellénistique. Cf. Lassus 1934 (n. 18, supra), p. 127-128.

22. En définitive, il n'est pas indispensable d'interpréter catégoriquement, optant ou non pour une signification allégorique; il suffit de constater la fréquence du sujet. Pour les chasses romaines, cf. A ymard 1961 (n. 20, supra), p. 469 sq., 574-575.

23. Cf. note précédente. Il faut évidemment tenir compte de la fréquence de ce genre de combats, de leur actualité lors de l'exécution de la mosaïque; cf. Cameron 1973 (n. 14, supra), p. 228-230, 242. Au sujet des venationes $\mathrm{cf}$. encore $\mathrm{L}$. Robert, Les gladiateurs dans I'Orient grec, Paris 1940, p. 309-331. Cf. A y mard 1961 (n. 20, supra), p. $54 \mathrm{sq}$

24. Cf. n. 16, supra.

25. Le terme en question, labouré par l'expérience et la pensée païenne, se fait donc traduire par des images à un moment qui ne coïncide pas avec sa propre "maturité" -qui leur est antérieure. Malgré la sérénité physionomique de la figure qui le personnifie, on soupçonne que ce terme est en quête de successeur, si l'on peut dire, et invisiblement pris dans un élan de fuite en avant - comme le sont toutes les fuites en histoire. L'on peut parallèlement comprendre que la version qui lui convenait dans cette œuvre du Ve siècle n'a pas marqué un point final dans l'évolution du vocabulaire pictural (cf. plus loin, Fig. 9). Du côté latinophone, une situation analogue est illustrée - mais sans recours à l'abstraction - dans une mosaïque de deux siècles plus ancienne, provenant de Smirat et actuellement conservée au Musée de Sousse. En dehors des venatores, y est figuré, entre autres, le dominus, Magérius, tandis que le milieu de la composition est occupé par un garçon qui présente les sommes dépensées pour le spectacle (cf. n. 20, supra). Il revient à Glanville Downey d'avoir suggéré la transition de la mégalopsychia païenne à la charité chrétienne: Downey 1945 (n. 18, supra), p. 283.

26. "La libéralité sied à la main droite impériale" nous signale Michel Italikos dans son discours à l'empereur Jean II Comnène (Lettres et

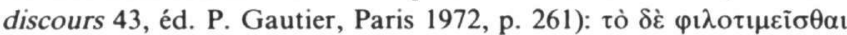

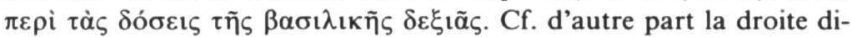
vine, réceptacle des âmes impeccables, dans la miniature du fol. 33 du psautier de Munich (ps. 23, 3; cf. Sagesse de Salomon 3, 1): Der serbische Psalter. Faksimile-Ausgabe des Cod. slav. 4 der Bayerischen Staatsbibliothek München. Textband unter Mitarbeit v. S. Dufrenne, S. Radojčić, R. Stichel, I. Ševčenko, Herausg. v. H. Belting, Wiesbaden 1978, p. 199-200. Cf. également la paume ouverte, versant de l'argent, de Juliana Anicia (Fig. 5): n. 31, infra. Sur le célèbre ivoire du Kunsthistorisches Museum de Vienne, l'augusta trônante, apparaissant, entre des rideaux ouverts, à l'intérieur d'un édicule qui l'abrite, emprunte le même geste de la paume ouverte face au spectateur, dans la version - l'avant-bras tendu à droite - que l'on avait précédemment attribuée à Constance II (Fig. 2), et que l'on a remployé pour figurer la libéralité de Juliana Anicia (plus loin: Fig. 5); Cf. Delbrueck 1929 (n. 15, supra), n 52; New York 1979 (n. 11, supra), $\mathrm{n}^{\circ} 25$ (Breckenridge). Pour l'identité de la personne figurée, cf. Ai $\kappa$.

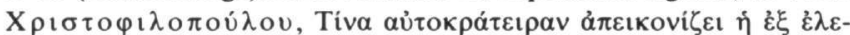

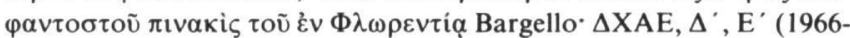
69), p. 146 sq. Pour le geste de Mégalopsychia, cf. Levi 1947 (n. 15, supra), p. 340.

27. Sur Ardabour, cf. n. 20, supra. Il est à noter que des auteurs chrétiens de la haute époque fustigaient ce gaspillage, en lui opposant la charité, qui, elle, n'est pas manifestée en public, mais devant Dieu et les anges. Cf. l'homélie pseudo-chrysostomienne sur Mt. 6, 1 sq.: PG 59, col. 572. Cf. également les remarques de L. Robert dans Hellenica 11-12 (1960), p. 569-573. Sur la différence entre l'évergétisme et la charité chrétienne, voir Veyne 1976 (n. 1, supra), p. 44-67; pour l'évolution de la générosité antique à la charité chrétienne cf. Patla gean 1977 (n. 1, supra), 181-196.

28. H. Gerstinger, Dioscurides, Codex Vindobonensis Med. Gr. 1 der Österreichischen Nationalbibliothek. Kommentarband zu der Faksimileausgabe, Graz 1970, p. 33 sq.; O. Mazal, Byzanz und das Abendland. Katalog einer Ausstellung der Handschriften- und Incunabelsammlung der Österreichischen Nationalbibliothek, 25 mai-10 octobre 1981, Vienne 1981, no 376 (p. 475-476), pl. coul. 17. Cf. encore D. Ainalov, The Hellenistic Origins of Byzantine Art, New Brunswick, N.J., 1961, p. 60-64, fig. 30; I. S path arakis, The Portrait in Byzantine Illuminated Manuscripts, Leyde 1976, p. 145-148, fig. 95. Cf. K. Weitzmann, Studies in Classical and Byzantine Manuscript Illumination, Chicago - Londres 1971, p. 154-155, fig. 132.

29. On peut voir l'effigie de l'époux de Juliana sur plusieurs diptyques consulaires; cf. surtout le diptyque conservé au Landesmuseum de Zurich, provenant de Constantinople, et datant de 506: Delbrueck, 1929 (n. 15, supra), n 9 (p. 110-111); W. F. Volbach, Elfenbeinarbeiten der Spätantike und des frühen Mittelalters, Mainz $1976^{3}, \mathrm{n}^{\circ} 8, \mathrm{pl}$. 4. Sur le diptyque de Zurich, Flavius Areobindus Dagalaifus préside aux jeux du cirque, figurés sur la moitié inférieure, sur deux registres superposés; au "fond" (au milieu, plus ou moins, des panneaux), l'on voit un plan superposé en hémicycle, avec une rangée de spectateurs. 30. Par l'instauration des deux équivalents latéraux (Magnanimité et Prudence), qui se balancent de part et d'autre de la princesse, cette image de majesté symbolique rejoint la classe iconographique des symétries trinaires. La même syntaxe des éléments compositionnels nous est bien familière par les diptyques consulaires; les deux capitales de l'Empire, Rome et Constantinople, qui très souvent sur les diptyques encadrent le consul "en fonction" dans l'une ou dans l'autre (cf. Volbach $1976^{3}$ - note précédente-, $\mathrm{n}^{\circ}$ 15, 24, pl. 4, 5), sont en l'occurence remplacées, dans le codex de Vienne, par des abstractions personnifiées, qui figurent les vertus du personnage loué.

31. Magnanimité porte en effet sur son sein, dans un pli de son manteau, un trésor de monnaies, dans lequel, le personnage historique puisse les pièces qu'il verse par la suite de sa paume ouverte. Cf. $n$. 26 , supra. Par ailleurs, des récipients identiques à celui de la mosaïque de Yaktô (Fig. 4), sont posés devant le podium sur lequel est hissé le siège de Juliana. Cf. n. 15, supra. A propos de la paume de Juliana, cf.

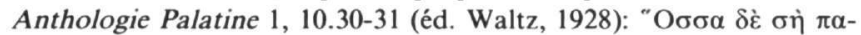

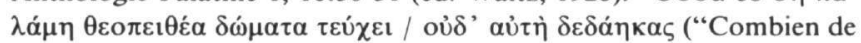
demeures où l'on sert Dieu construit ta main, tu ne le sais pas toimême").

32. La prostration de la Reconnaissance des arts traduit également une différence de niveau d'être entre la donatrice et les figures qui personnifient son appareil moral d'une part, et d'autre part les arts qui en bénéficient; les arts eux-mêmes ne servent d'ailleurs qu'à l'échafaudage d'un décor rayonnant et périphérique. Un autre moyen pictural pour traduire une différence de condition, consiste peut-être en l'altérité de style, qui distingue la gravité palatiale de la figure médiane, de la fraîcheur et des poses décontractées des personnages latéraux. Cf. Weitzmann 1971 (n. 28, supra), p. 154-155; S patha rak is 1976 (n. 28, supra), p. 147-148 (Weitzmann distingue entre la miniature de dédicace et le portrait d'auteur du fol. $4 \mathrm{v}$; cette remarque est transposée au sein de la même composition par Spatharakis).

33. Cf. Théophane, Chronographie, Bonn, 1839, t. 1, p. 243.7-9:

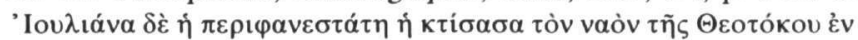

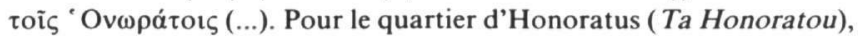
cf. R. Janin, Constantinople byzantine. Développement urbain et 


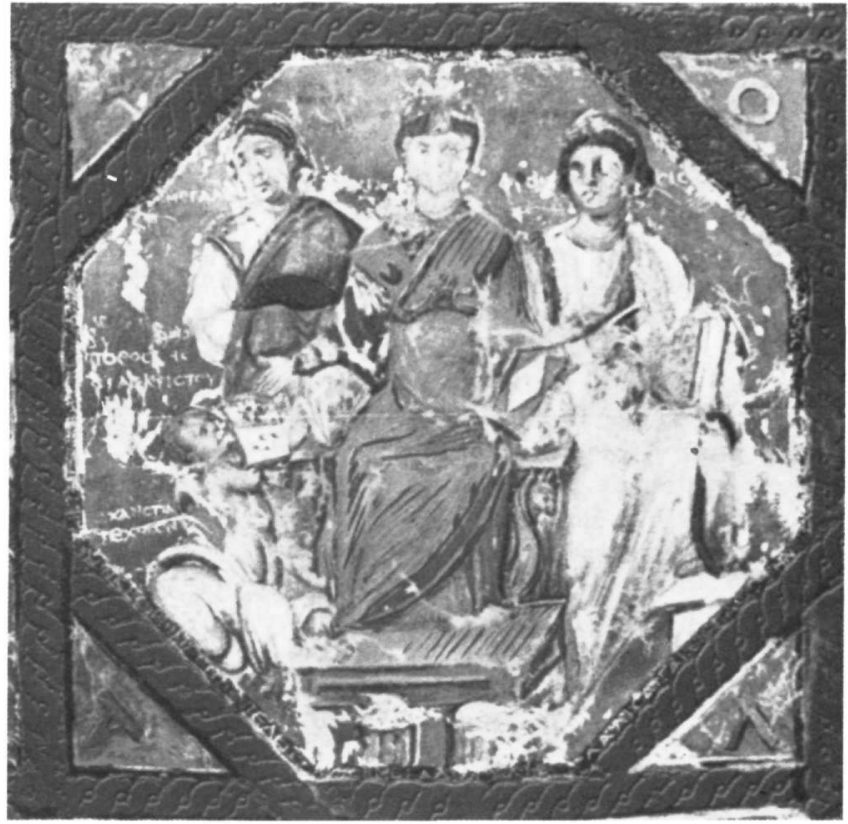

Fig. 5. Vienne, Bibl. nationale. Cod. med. gr. 1 (Dioscoride). Déb. VIe s. Fol. 6v: Juliana Anicia entre Mégalopsychia et Phronèsis

l'empereur est représenté comme constructeur d'églises ${ }^{35}$; il figure désormais, en donateur, à côté du Christ $^{36}$, ou de la Théotocos ${ }^{37}$, portant la bourse avec l'argent sacralisé ${ }^{38}$. Un souci d'équilibre arétologique (prudence tempérant magnanimité), en même temps qu'un desideratum compositionnel, appuyé sur des antécédents modeleurs, ont sans doute présidé au redoublement des parèdres. Mégalopsychia tient toutefois la place d'honneur, comme plus tard la Miséricorde placée à la droite du Christ ${ }^{39}$. Phronèsis, sa collègue, est dotée d'une ample postérité dans le domaine des images ${ }^{40}$; par contre, pour ce qui concerne Mégalopsychia, nous constaterons que l'image du fol. $6 \mathrm{v} \mathrm{du}$ codex de Vienne témoigne de sa dernière apparition.

En définitive, la composition du Dioscoride de Vienne illustre une féconde et rayonnante justesse d'être; topos laudatif destiné à des répercussions fréquentes, on le sait, mais qui gagne l'optimum de concision par la symétrie trinaire que nous projette le langage pictural. La rectitude de la personne médiane, prolongée de surcroît par le iota de l'initiale de son nom, inscrit sur le triangle de pourtour qui la surmonte ${ }^{41}$, et de part et d'autre, la vague ondulation des figures latérales, suggèrent la mécanique d'une balance à deux plateaux. On obtient par ailleurs une image ressamblante avec le portrait moral que Michel Italikos, au XIIIe siècle, compose de l'ex-basilissa Irène Doukaina ${ }^{42}$, dont il exalte l'état symétrique de l'âme ${ }^{43}$.
Ce n'est plus en ouvrant la main droite, mais en comptant presque les pièces données, qu'est représentée H KYPA CI $\Lambda \Theta O Y C$ (Fig. 6), fixée dans une attitude solennelle, sur une mosaïque de pavement récemment découverte en Israël ${ }^{44}$. Ce panneau figuré, datant du VIe siècle (578 ?), est placé à l'intérieur d'une basilique à trois nefs, près de l'entrée, dans le deuxième intervalle de l'entrecolonnement nord qui sépare la nef centrale du bas-côté gauche. La signification du geste est évidente, mais l'objet concret de la donation nous échappe: l'argent était-il dépensé pour l'exécution des mosaïques, la construction de l'église, ou la fabrication des vases sacrés? Il en est ainsi également pour l'identité de la partenaire plus âgée de Silthous, au visage osseux, que l'on voit à gauche, présentée aussi, comme la donatrice, en buste et de face. Le riche apparat de son costume indique qu'il ne s'agit pas d'une servante, mais d'une personne de haut rang; mais quel peut être son statut ontologique? Figure-t-elle discrètement l'Eglise? Elle tient ostensiblement un récipient ${ }^{45}$ : s'agit-il d'un vase sacré? Et de quoi exactement ce récipient est le support? Si l'on suppose que l'inscription KA $\Lambda H \Omega P A$ ne se rapporte pas à l'identité personnelle de la figure de gauche ${ }^{46}$, peut-on admettre qu'elle suggère l'accueil que cette femme réserve à Silthous, en recevant sa donation? Une lecture plus avancée, en passant par le témoignage de l'image de la philoktistos Juliana (Fig. 5), devra être appuyée sur des documents contemporains -à la fois épigraphiques et iconographiques - provenant de la même région.

Comme elle adhère à l'idéologie des donations recréatrices, il faudra, parmi les oeuvres dont l'iconographie se rattache à notre thématique, réserver une place à la composition monumentale de la mosaïque de pavement de Qasr el-Lebya ${ }^{47}$. Des nombreuses unités iconographiques sont ici réparties dans des panneaux séparés, symétriquement disposés. Un des panneaux médians s'ouvre sur une vue frontale d'un édicule cultuel abritant l'image d'Ananéôsis (ANANESCIC: Rénovation), qui apparaît entre des rideaux ouverts (Fig. 7) ${ }^{48}$. Le récipient (apparemment un panier), débordant d'objets sommairement définis, que la figure allégorique tient sur sa gauche, nous fait penser à la sporta que tenait la Magnanimité de Yaktô (Fig. 4) ${ }^{49}$; or, le geste des deux figures n'est pas identique. Ces deux versions, dont la seconde est d'un siècle plus récente que la première, correspondent dans leur "mécanique"; elles inaugurent pourtant des opérations motivées chaque fois par des intentions différentes, et par conséquent, elles nous renseignent sur la variété des possibilités typologiques que peuvent recouvrir des formations iconographiques ressamblantes ${ }^{50}$.

Discrètes -mais dans un milieu suffisament bien 
répertoire topographique, Paris $1964^{2}$, p. 486. Pour les diverses ktiseis de Juliana Anicia cf. Anthologie Palatine 1, 10; ibid., 12-17. L'épigramme 10 figurait sur le décor sculpté de l'église de Saint Polyeucte; c'est d'ailleurs en lisant certains passages (parmi lesquels, partiellement, le passage cité plus haut, n. 31) de cette épigramme sur des fragments retrouvés de ce décor, que Cyril Mango et Igor Ševčenko ont pu reconnaître les vestiges de ce monument constantinopolitain: C. Mango - I. Ševčenko, Remains of the Church of St. Polyeuctos at Constantinople, DOP 15 (1961), p. 243-247. D'autre part, au sujet de la magnanimité du bâtisseur d'églises, cf. une attribution analogue que nous procure Grégoire de Nazianze, dans l'oraison funèbre qu'il

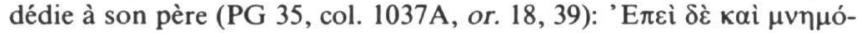

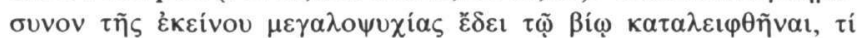

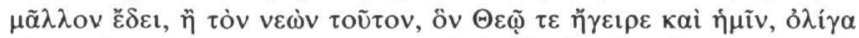

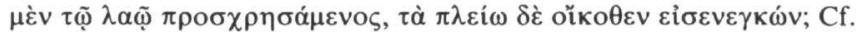
Pat lagea n 1977 (n. 1, supra), p. 197, n. 310 (d'autres exemples, ibid., n. 311). Pour l'épigramme du codex de Vienne, cf. Gerstinger 1970 (n. 28, supra), p. 34 .

34. Empruntant à la novelle 105 de Justinien, qui attribue à l'empereur la permanence du consulat (diènékès hypateia: cf. n. 59, infra), on pourrait qualifier de ktistès diènékès le souverain byzantin de haute époque: fondateur/rénovateur permanent de la cité chrétienne dans son ensemble, et toujours dans un esprit d'aménagement urbain antique. Notons que vaillance, magnanimité et amour de faire bâtir, sont

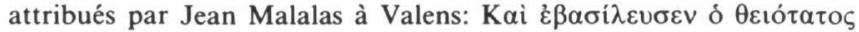

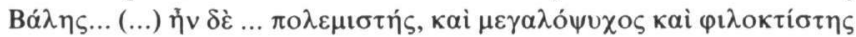
(Chronographie, Bonn, 1831, p. 342). On ne saurait préciser si la grandeur d'âme du mégalopsychos se rapporte, dans ce cas, plutôt à l'amour de bâtir du philoktistos qu'à vaillance martiale du polémistès. Cf. Patlagean 1977 (n. 1, supra), p. 196-197. Pour les valeurs sémantiques du verbe ktizein et du substantif ktistès attribués à la personne de l'empereur, cf. la notice substancielle que nous fournit G. Downey, Imperial Building Records in Malalas, BZ 38 (1938), p. 10-11, n. 3. 35. Cf. V. Lazarev, Storia della pittura bizantina, Turin 1967, fig. 157; A. Grabar, La peinture byzantine, Paris $1979^{2}$, pl. p. 95.

36. Lazarev, ibid., fig. 161; Gravar, ibid., pl. p. 98.

37. Lazarev, ibid., fig. 289; Grabar, ibid., pl. p. 99.

38. Cf. N. Oikonomidès, The Mosaic Panel of Constantine IX and Zoe in Saint Sophia, REB 36 (1978), p. 219-232.

39. Grabar 1936 (n. 20, supra), pl. 24.2; Lazarev, ibid., fig. 251. 40. Elle est figurée dans deux manuscrits, dont il sera question plus loin (cf. n. 74-75, infra). Cf. H. Bu ch th a l, An Illuminated Byzantine Gospel Book of about 1100 A.D., Special Bulletin of the National Gallery of Victoria, Centenary Year 1961, Melbourne 1961, fig. 5. Elle est également figurée, avec Andreia, sur les battants de la porte d'une pièce d'orfèvrerie en forme d'édicule, conservée dans le Trésor de Saint-Marc. Cf. Venise, Palazzo Ducale, 1974, Venezia e Bisanzio, $n^{\circ}$ 44.

41. Cf. Flavius Cresconius Corippus, Iust. 1, 353: l'empereur Justin, sacrum sic iota resurgens. Les lettres qui composent le nom de la princesse sont inscrites, chacune séparément, dans les secteurs triangulaires du pourtour.

42. Il s'agit de la mère de l'empereur Jean II Comnène, dont l'épouse portait d'ailleurs le même nom.

43. Michel Italikos, Lettres et discours 15, éd. Gautier (n. 26,

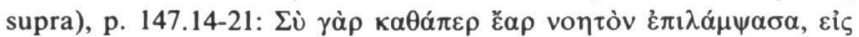

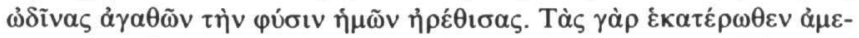

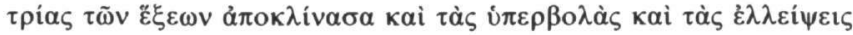

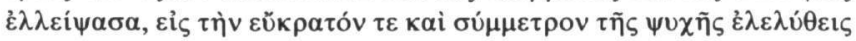

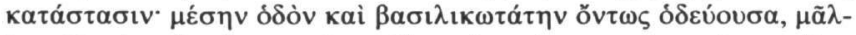

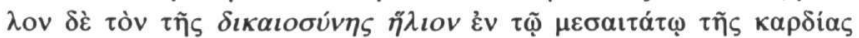

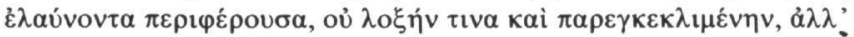

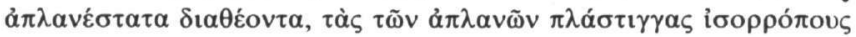

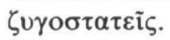

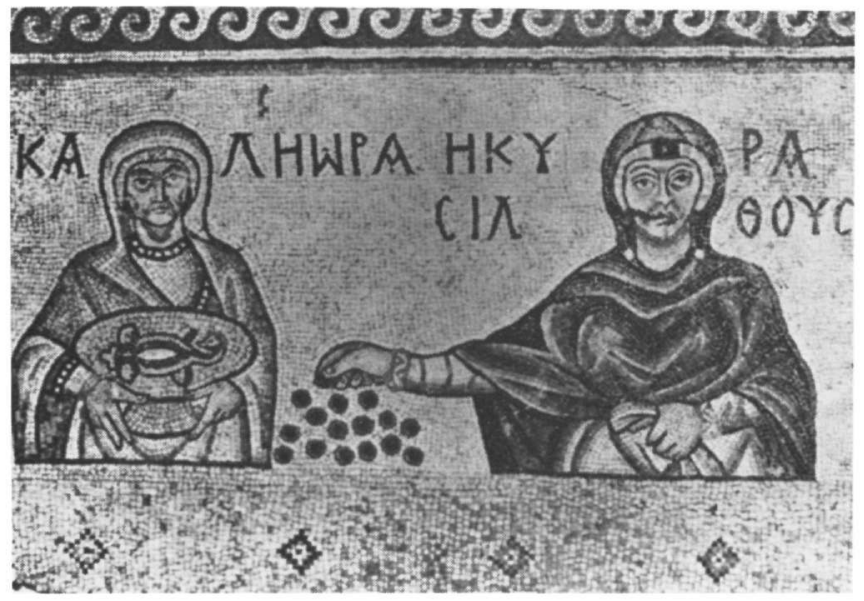

Fig. 6. Kissoufim. Mosaïque de pavement. VIe s. Panneau de Silthous

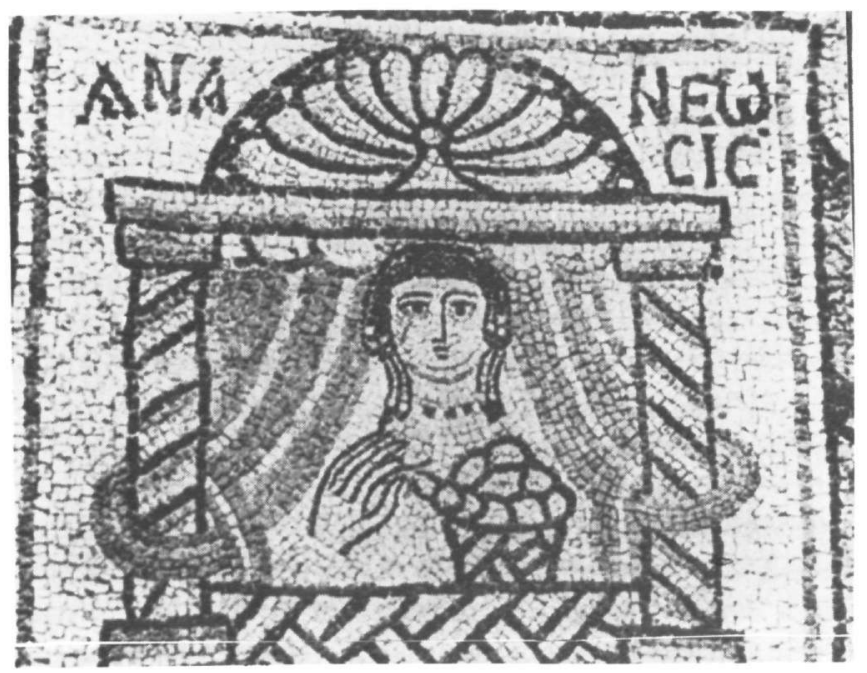

Fig. 7. Qasr el-Lebya, Eglise est. Mosaïque de la nef (dét.): I'Ananéôsis. VIe s.

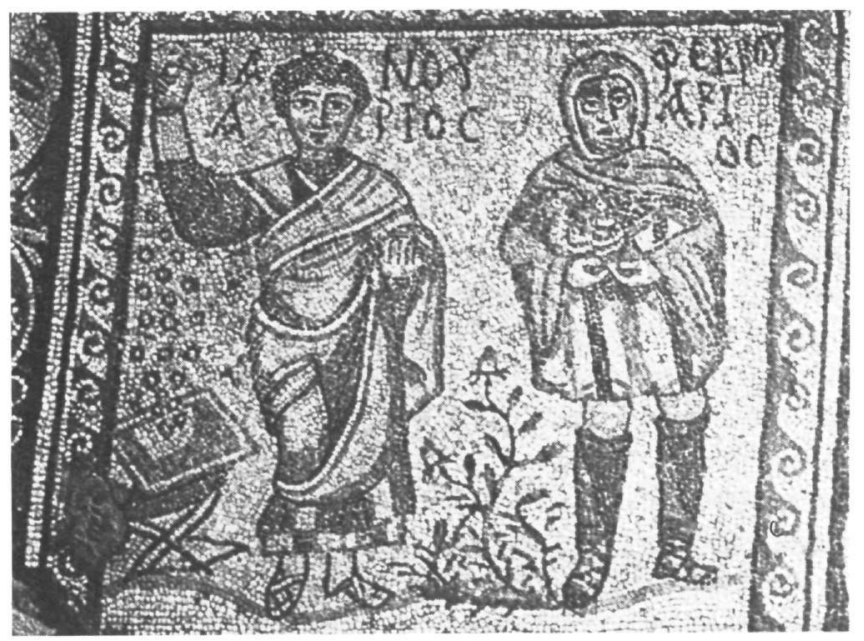

Fig. 8. Argos, Musée archéologique. Mosaïque de pavement. Vers 500? Janvier, en consul, répandant des monnaies 
défini- projections de l'eidos de la vertu de libéralité d'une personne évanescente (Fig. 4) ${ }^{51}$, ou explicites, bien que secondaires, attachées auprès du personnage agissant (Fig. 5), les apparitions de Mégalopsychia n'épuisent pas le répertoire des transpositions prosopographiques, des mutations de niveau d'identité, des aller retour entre la personne historique "en fonction" et la fonction personnifiée, dans la mise en scène de la sparsio; nous l'avons déjà vu à propos de Constance II versant des monnaies, dont l'image dans le Calendrier de 354 (Fig. 2) est en elle-même le lieu mutateur, au sein duquel, d'une part, le concret du personnage frise l'abstraction de son rayonnement rituel, et d'autre part, vice versa, l'abstrait rituel s'avère une des dimensions constituantes du représenté.

C'est ainsi qu' une figure impersonnalisée de consul, vu debout et portant la toga, sert de terme pictural pour personnifier le mois de janvier, qui, accompagné de Février (Fig. 8), dans le même cadre, s'inscrit dans la bande des panneaux d'un pavement en mosaïque (vers 500 ou plus tard?) figurant les mois, le long du portique sud d'une villa à $\operatorname{Argos}^{52}$. Le signe donné avec la main droite, tenant la mappa, pour le commencement - suppose-t-on- des jeux du cirque (tel qu'il est d'habitude figuré sur les diptyques consulaires), ainsi que la sparsio, opérée par le moyen de 23 pièces d'or qui s'écoulent de la même main levée, dépendent dans cette ouuvre du même geste.

Le personnifiant reflète sans doute les "souhaits" des spectateurs: souhaits formulés par l'évocation de temps forts d'une ambiance publique, qui transpose la pensée, de la région rurale où se situe cette villa, en l'espace d'une métropole. Le coup de départ annuel est donné en cité, tandis que la relève est ensuite assurée par les revêtements successifs des campagnes - représentées par les mois ${ }^{53}$. Cette image de consul semble avoir égalé dans les consciences celle d'un "automate" déclenchant le bonheur. Mais il faudrait envisager de quelle conscience on parle; celle du propriétaire de la villa? Aussi, il se pourrait que celui-ci entendît s'y reconnaître personnellement; ou bien, il voulait évoquer la dignité par laquelle on avait naguère honoré un membre de sa famille. Les hypothèses peuvent fourmiller puisque l'identité historique du personnage nous est inconnue; elles n'en deviennent pas plus utiles pour notre propos, où nous retiendrons toutefois ce phénomène d'oscillation entre la personne en fonction et la fonction personnifié $\mathrm{e}^{54}$.

Concrètement répétitif, le geste de la semence monétaire, ayant accédé à la pérennité de l'imaginaire, se cristallise sur la mosaïque d'Argos, dont les éléments mis en scène, attribués à la personne "fécondante" du consul, instituent les conditions d'une dynamique de départ, qui se développe par la suite en épisodes "automatiques" 55 . M̀̀v í $\pi \dot{\tau} \omega v \pi \rho \tilde{\tau} \tau \zeta$ ("le mois des consuls vient le premier") nous enseigne l'épigramme 580 de l' Anthologie Palatine 956; et le premier des mois emprunte le costume des consuls. Quand l'éclat de l'abondance disparaît avec le primat de la dignité57, le vocabulaire persiste, désormais réactivé par les largesses irrégulières des basileis de la moyenne période ${ }^{58}$.

Le consul (fictif) d'Argos était antérieur à Justinien,

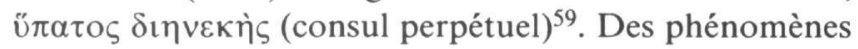
du même ordre que ceux dont témoignent les images étudiées jusqu'ici, s'inscrivent dans la conjoncture immédiatement post-justinienne. Flavius Cresconius Corippus les enregistre dans le "panégyrique" qu'il rédige à l'occasion de l'accession de Justin II (565-578), neveu de l'empereur défunt (mort le 14 novembre 565), et sur l'inauguration de l'année par le nouvel empereurconsul (1er janvier 566) ${ }^{60}$. Le témoignage de cet auteur est doublement important. Certaines données, désormais constantes, de l'idéologie impériale, sont formulées en termes de latinité byzantine; et ceci s'effectue dans un temps où l'héritage antique se désintègre. Ce document récapitulatif du patrimoine, reflète en même temps le projet (rétrospectivement) novateur du nouveau maître au pouvoir.

Exalté par Corippus, l'empereur se trouve à un niveau intermédiaire entre Dieu et son peuple. L'étagement hiérarchique n'est pas inconnu. D'autres comptables de la construction des pouvoirs avaient auparavant établi des définitions appropriées, et en $\operatorname{grec}^{61}$. Le texte de Corippus reste pourtant, de notre point de vue, un lieu privilégié. Les vertus qui transcendent le personnage particulier, le liant à Dieu et au peuple chrétien, sont la pitié et la clémence; et la pratique de la clémence assimile l'empereur au Christ, dont il devient l'imago. Concentrons notre attention sur ces événements qui se rattachent à la dispersion augurale, à la distribution généreuse. La paix civile, gardée par les citoyens, sera rétribuée par la générosité impériale, annoncée par Justin au peuple ${ }^{62}$; il se voulait, rappelons-le, restaurateur des anciens fastes. Le peuple se réjouit à l'annonce des largesses à venir ${ }^{63}$. Deux épisodes mettent particulièrement en relief les qualités du nouveau souverain; l'éclat de ses “énergies" éblouit la masse expectante, qui s'agite dans l'attente des nouvelles manifestations de la générosité impériale. L'annulation des dettes sert de preuve de la pietas/pitié de l'empereur ${ }^{64}$, qui avait auparavant libéré les prisonniers ${ }^{65}$. Autrement plus efficaces qu'une captatio benevolentiae rhétorique, les mesures impériales, interprétées comme des ouvres de piété66, forcent l'adhésion des dignitaires et de la mouvence populaire. Les schémas sont assumés avec vigueur. Pieux-miséricordieux (v. 402) et clément-juste (v. 423), image du Christ 
44. R. Cohen dans Qadmoniot 12 (1979), p. 19 sq.; A. Ovadiah S. Mucznik, The Pavement of Kissufim, Mosaïque. Recueil d'hommages à Henri Stern, Paris 1983, p. 273-280; cf. M. Mango, Silver from Early Byzantium. The Kaper Koraon and Related Treasures, Baltimore 1986, p. 11, fig. I.2.

45. "The inner top part of the vessel is decorated with a bird which is difficult to identify and is presented in a stylized, flat way": Ovadiah-Mucznik, ibid., p. 275. Un banquet fut-il probablement donné à l'occasion de l'inauguration de l'oeuvre exécutée?

46. Cf. ibid., p. 276.

47. A. Grabar, Recherches sur les sources juives de l'art paléochrétien, CahArch 12 (1962), p. 135-140; id., Une nouvelle interprétation de certaines images de la mosaïque de pavement de Qasr el-Lebya ( $\mathrm{Li}$ bye), Comptes rendus de l'Académie des Inscriptions et Belles-Lettres, 1969, p. 264-279; H. St er n, Quelques remarques sur les mosaïques de Qasr el-Lebya, Comptes rendus... (cf. supra), avril-juin 1969, p. 279282; M. Guarducci, La più antica catechesi figurata: il grande musaico della basilica di Gasr Elbia in Cirenaica, Atti dell'Accad. Naz. dei Lincei, Classe di Scienze morali, storiche e filologiche, Memorie, 18 (1975), p. 659-686; S. Dufrenne, L'Ananeôsis de Qasr el-Lebya, Antiquités Africaines 16 (1980), p. 241-249; E. Alföldi-Rosenbaum - J. Ward-Perkins, Justinianic Mosaic Pavements in Cyrenaican Churches, Rome 1980, p. 34-37. Pour les valeurs sémantiques du mot ktisis, cf. n. 34, supra.

48. Immédiatement au-dessus du panneau d'Ananéôsis, on peut voir une vue "frontale" et symétrique de la "Ville Néa Théodôrias", flanquée sur ses côtés par les personnifications de la kosmèsis (décoration) et de la ktisis (fondation), inscrites dans des panneaux indépendants. Cf. Levi 1947 (n. 15, supra), p. 248-256, 349-350 (Ananéôsis, Ktisis, et autres personnifications), 320-321 (Ananéôsis), 347, 357-358 (Ktisis); cf. Dunbabin 1978 (n. 20, supra), p. 253-256; ibid., p. 228 n. 146. Voir en dernier lieu Alföldi-Rosen ba um - Ward-Perkins, ibid., p. 34-37, pl. 53; ibid., p. 40 sq. En rapport avec l'édicule dans lequel s'inscrit l'apparition d'Ananéôsis (Fig. 7), cf. la mise en scène de Constance II dans le Calendrier de 354 (Fig. 2), ainsi que l'augusta trônante de l'ivoire de Vienne: n. 26, supra.

49. L'apparence peut être trompeuse, dans la mesure où elle nous empêche de discerner ce que le commanditaire entendait faire représenter plus précisément; celui-ci devait en tout cas composer avec les moyens dont on pouvait disposer sur place; d'où, probablement, le rendu approximatif. Au sujet des sportae, cf. n. 15, supra. Sur le panneau de Qasr el-Lebya, Ananéôsis tend la main droite vers ce "panier", pour en détacher un des objets qui y sont contenus.

50. En vue de préciser certains détails, il faudra peut-être reprendre le dossier de l'Ananéôsis de Qasr el-Lebya. Cf. l'interprétation récente de H. Maguire, Sign and Symbol in the Nave Mosaic of the East Church at Qasr el-Lebya: A Case Study, Byzant. Stud. Conference, Abstracts of Papers 9 (1983), p. $72-73$ (cet auteur met l'accent sur la présence de Ktisis).

51. Surtout pour des lecteurs imprévus et postérieurs. D'autre part, la personna virtutis gagnait son autonomie après la disparition de la personne physique.

52. G. A kerström-Hougen, The Calendar and Hunting Mosaics of the Villa of the Falconer in Argos. A Study in Early Byzantine Iconography, Stockholm 1974, p. 23, 73-74; fig. 9.1; pl. coul. 1.1. Pour la datation, cf. ibid., p. 71, 152. Cf. encore R. Ginouvès, La mosaïque des mois à Argos, BCH 81 (1957), p. 216-268; Stern 1953 (n. 10, supra), p. 157, 222-223, pl. 32.1; C.-O. Nordst röm, Some Iconographical Problems in the Argos Mosaics, CahArch 26 (1977), p. 73-80. 53. Cf. A kerström-Hougen, ibid., fig. 9-11, pl. coul. 1-3.

54. Cette apparition du mois personnifié en guise de janvier n'est pas unique. Elle se retrouve en effet sur le Zodiaque du Ptolémée de la Vaticane (Bibl. Vaticane, cod. gr. 1291, fol. 9). Cf. Akerström-
Hougen, ibid., p. 73; I. Spatharakis, Some Observations on the Ptolemy Ms. Vat. Gr. 1291: Its Date and the Two Initial Miniatures, BZ 71 (1978), p. 46, pl. 6.

55. Ce sont les "épisodes" qu'enchaîne la course des mois.

56. Cf. Akerström-Hougen, ibid., p. 73.

57. Cf. la novelle 105 de Justinien: Corpus juris civilis, 4, Novellae, éd. Schoell - Kroll, 1912, p. 500-507; ibid., p. 503.30-504.14: le chrysion riptein (p. 503.30) est réservé à l'empereur. Cf. la novelle 94 de Léon VI: P. Noailles - A. Dain, Les novelles de Léon VI le Sage, Paris 1944, p. 308-311 (De l'abrogation de la loi sur le consulat); ibid., p. 311.15-22 (texte français, p. 310): “...maintenant, comme le cours du temps a modifié toutes choses et a fait passer cette grandeur consulaire de la gloire et de la splendeur ancienne à une condition modeste, et comme ceux qui y accèdent non seulement ne sont plus assez riches pour faire des libéralités aux autres, mais encore ne peuvent même pas se suffire à eux-mêmes, attendu que, par suite, la loi sur le consulat a été ensevelie dans un silence profond, ... nous écartons cette loi de notre legislation,...".

58. Sur le consulat, cf. R. Guilland, Recherches sur les institutions byzantines, 2, Berlin - Amsterdam 1967, p. 44-67. Rappelons qu'en plein XIVe siècle, lors du couronnement d'Andronic III Paléologue, des épikombia (bourses) sont jetés du haut des dégrés du socle de la statue équestre de Justinien. Cf. Pseudo-Kodinos, Traité des Offices, éd. J. Verpeaux, Paris 1966, p. 255.1-19, 271.1-24, 360.19-361.3. Pour le même geste, réactivé lors du sacre de Henri II, en 1547, cf. R. A. Jackson, De l'influence du cérémonial byzantin sur le sacre des rois de France, Byzantion 51 (1981), p. 203 sq. Sur les largesses plus récentes, cf. Hendy 1985 (n. 14, supra), p. 196 sq.

59. Cf. la novelle de Justinien, citée supra, n. 57, éd. Schoell - Kroll, p. 507.10-11.

60. Voir l'Eloge de Justin II, de Corippus, dans l'édition d'Averil Cameron (n. 15, supra). Cf. l'édition française de S. Antès, Paris 1981. 61. Cf. l'Ekthésis d'Agapètos, PG 86, col. 1163-1186; cf. P. Henry, A Mirror for Justinian: The Ekthesis of Agapetus Diaconus, GRBS 8 (1967), p. 281-308.

62. Des jeux à l'Hippodrome et des largesses consulaires lui seront offerts; Iust. 2, 348-356, éd. Cameron (n. 15, supra), p. 58 (cf. la traduction, ibid., p. 101): si socium socius, si civem civis amabit, / non solum optati jubeo spectacula circi, / praemia sed populis et maxima dona parabo. / ditabo plebes opibus, nomenque negatum / consulibus consul post tempora tanta novabo, / gaudeat ut totus Iustini munere mundus. / dona calendarum properant vicina mearum; / vos vestris adstate locis, properate, parate, / promisaque die nostras sperate curules.

63. Ibid., 357-360. Cf. le commentaire de Cameron, p. 175.

64. Cf. ibid., 402: dominus servis miseratus egenis. Cf. le psaume 40, 2 et son illustration dans les psautiers "à illustrations intégrales": $\mathrm{S}$. Dufrenne, Tableaux synoptiques de 15 psautiers médiévaux à illustrations intégrales issues du texte, Paris 1978. Pour l'illustration correspondante du psautier d'Utrecht, cf. ead., Les illustrations du psautier d'Utrecht. Sources et apport carolingien, Paris 1978, pl. 31.12, 38.6. Dans cette image, les pauvres, mentionnés par le texte illustré, sont assistés par le "bienheureux" qui les nourrit. C'est exactement le super egenum et pauperum du verset 2 qui déclenche le mécanisme morphopoétique; tandis que le beatus vir, tout en renvoyant à la béatitude, ne la convoque pas se ipsam. L'absence d'écho dans les psautiers grecs du XIe siècle, est suivie par l'apparition d'une scène dans leur progéniture du XIe, qui reflète des préoccupations contemporaines (cf. l' "irruption de saints" dans l'illustration des psautiers du XIe siècle); cf. S. Der Nersessian, L'illustration des psautiers grecs du moyen âge, II. Londres Add. 19352, Paris 1970, p. 30, fig. 83. Dans le Sinaïticus 48 (fol. 50), de l'illustration du même verset n'est préservée que la figure du bénéficiaire; Kurt Weitzmann (Byzantine Litur- 


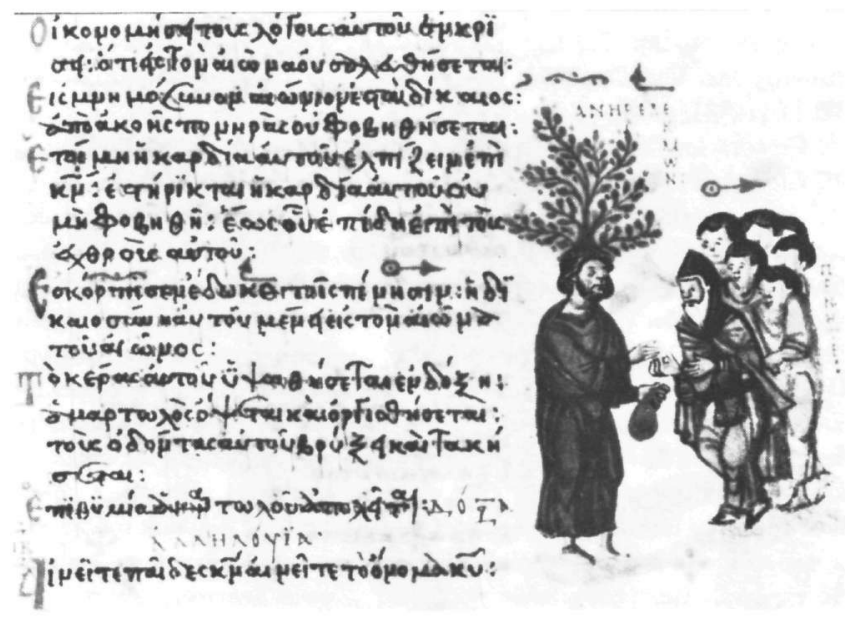

Fig. 9. Moscou, Musée historique. Cod. gr. 129 (psautier Chludov) IXe s. Fol. 116 (ps. 111, 9): le miséricordieux

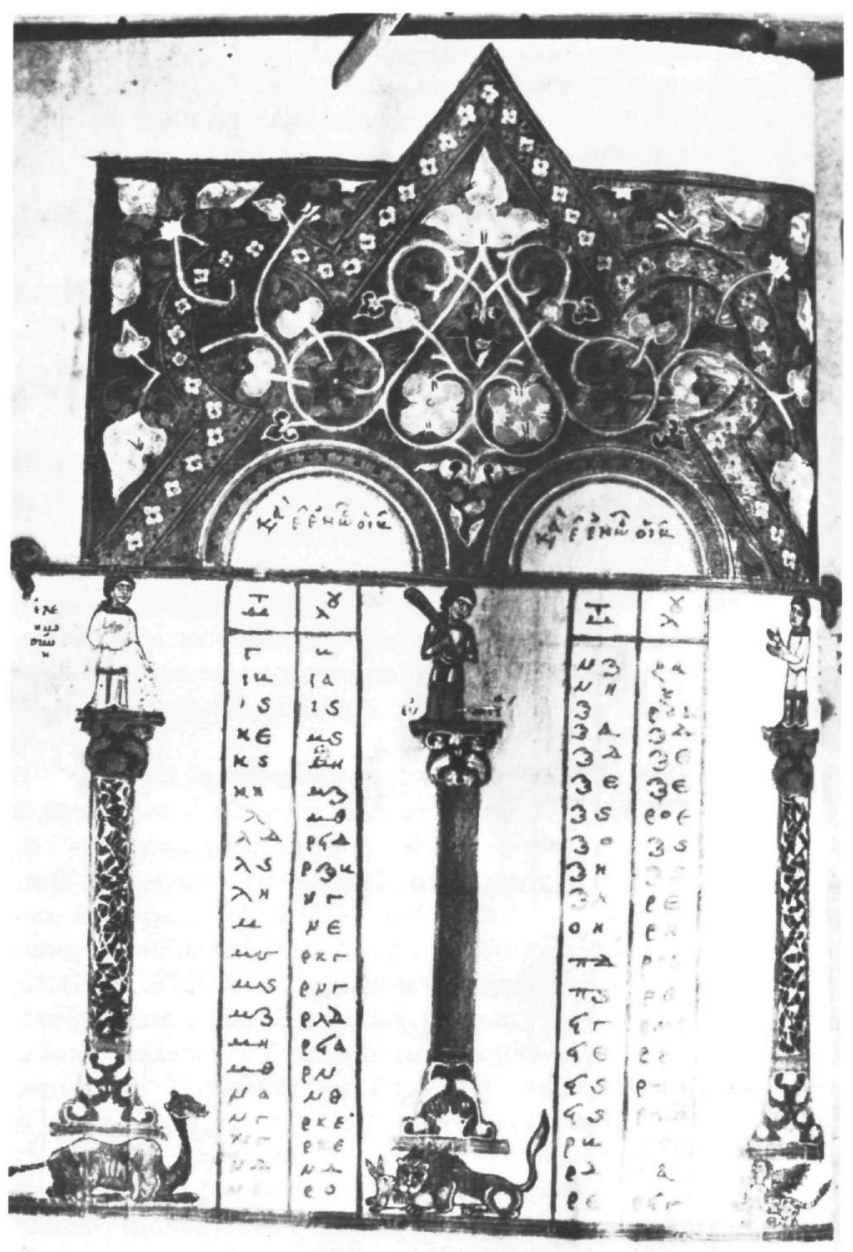

Fig. 10. Venise, Bibl. Marcienne. Cod. gr. Z 540 (Evangiles). Déb. XIIe s. Fol. 5v: Tables de canons (v. 428), Justin incarne le concept de philanthropia ${ }^{67}$. Le premier janvier est arrivé. Les dona calendarum (v. 103), seront distribués aux dignitaires dans le palais ${ }^{68}$. Les officiers de service sportisque capacibus aurum adcumulant (v. 105-106) ${ }^{69}$. Les sénateurs (v. 142 sq.) reçoivent les cadeaux distribués par l'empereur (v. 148 sq.), comme les paysans cueilleraient les produits de la terre. L'auteur se souvient également du champ natal de ses expériences; même le ciel ne manque pas dans la comparaison. Au dehors du palais, les regards du peuple sont fixés sur les portes royales (v. 213-214), attendant l'apparition de l'empereur-consul: c'est d'une façon comparable qu'en Libye, les laboureurs, attendants que la pluie annoncée par des coups de tonnerre inonde la terre sèche, se tournent vers le ciel traversé par des éclairs ${ }^{70}$. Il devient évident que dans cet enregistrement de la projection du consul dans l'espace public, nous pouvons suivre dans leur déroulement toutes les étapes qui précèdent l'épiphanie bénéfique du souverain.

Le geste ostentatoire, aveugle ou réfléchi, disparaît (et de la pratique, et du domaine des images); le vocabulaire persiste, mais il relève désormais d'une figure rhétorique $^{71}$. Sainte Thomaïde est ainsi présentée par

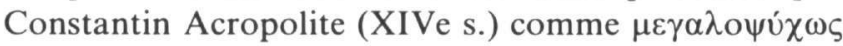
ă $\gamma \alpha \nu$ $\sigma \kappa о \rho \pi i \zeta o v \sigma \alpha$ (dispersant avec une extrême magnanimité $)^{72}$. Or, la demonstration picturale chrétienne vise l'autre personnellement (de la main à la main). Mais pour qu'il y ait aumône, il faut qu'il y ait des pauvres; et cette catégorie sociale ne s'impose dans toute la clarté de ses contours que dans la conscience chrétienne. S'instaure ainsi une polarité coopérante. Et face au Rédempteur, tous les humains ne sont que des pauvres. Par conséquent, avec ceux qui bénéficient de l'assistance du prochain, il y a aussi ceux qui ont besoin de la parole de Dieu ou de l'exemple du juste.

Cependant —étant donné l'écart chronologique qui existe souvent entre la propagation d'une idée, ou d'une pratique, et leur traduction plastique-, pour qu'il y ait formation d'une image, il faut que la conscience de la société soit travaillée dans des conditions particulières, et dans le but d'une démonstration qui, à ses débuts, peut même revêtir des allures polémiques. Les pauvres, comme d'ailleurs le rapport qui les relie à leurs bienfaiteurs, les justes, ne sont en effet figurés en tant que tels qu'au IXe siècle, au lendemain de l'Iconoclasme, quand l'image de Dieu réapparaît dans l'iconographie et sur les monnaies. L'aumône picturale confirme ainsi la foi orthodoxe. Présents dans les psautiers à illustrations marginales (Fig. 9), ils sont répercutés ultérieurement dans des illustrations de textes patristiques ou hagiographiques, notamment dans des illustrations de l'homélie $\Pi \varepsilon \rho i ̀ ~ \varphi \imath \lambda o \pi \tau \omega \chi i ́ a \varsigma$ de Grégoire de Nazianze ${ }^{73}$.

Enfin, pour ce qui est de l'image d'un personnage qui 
répand de l'argent, elle est recréée aux XIe-XIIe siècles, sur les tables de canons de deux tétraévangiles, conservés respectivement à la Bibliothèque Marcienne (Venise) ${ }^{74}$ et à la National Gallery of Victoria (Melbourne $)^{75}$. Il s'agit maintenant de personnages minuscules, supportant, avec d'autres partenaires, les arcs bilobés qui encadrent les tables de canons (Fig. 10). Or ce geste ne renvoie plus à un événement. La figure allégorique qui le mime ne s'appelle plus Mégalopsychia, ver-

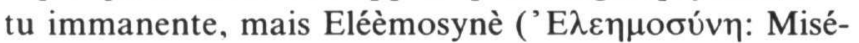
ricorde), propriété de Dieu imitée par les humains. Le geste en question revêt ainsi une valeur quasi intemporelle, dont la vigueur est appuyée sur le geste - sûrement intemporel-du semeur ${ }^{76}$. Et dans l'horizon de l'imaginaire chrétien, le semeur archétypique, le plan-

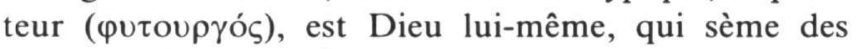
"verbes" éveilleurs sur la terre rationnelle, la raison humaine. Voici pourqoi, à partir du IXe siècle, on voit le miséricordieux portant sur sa tête un arbre (Fig. 9) ${ }^{77}$ : la semence divine a germé!

ELIAS ANTONOPOULOS

\section{Origine des photographies}

Fig. 1: Photo Anderson, Rome. - Fig. 2: Stern, 1953 (n. 10, supra), pl. 14. - Fig. 3, 5, 7, 9-10: Collection chrétienne et byzantine, Paris (Ecole pratique des Hautes Etudes, Section des sciences religieuses). - Fig. 4: Levi, 1947 (n. 15, supra), vol. 2, pl. 76b. - Fig. 6: Cohen, 1979 (n. 44, supra). - Fig. 8: Akerström-Hougen, 1974 (n. 52, supra), pl. 1.1.

gical Psalters and Gospels, Londres 1980, art. VII, p. 5-6) se demande si la figure manquante était celle du Christ; mais était-ce possible que le Christ soit représenté donnant de l'argent? Rapellons que les distributions de vivres du psautier d'Utrecht (Dufrenne, citée supra, p. 103-105), correspondent dans les psautiers grecs à des scènes d'aumône.

65. Ibid., $361 \mathrm{sq}$.

66. Ibid., 407. Cf. l'Ekthésis d'Agapètos (Exp. cap. admon. 48, PG 86,

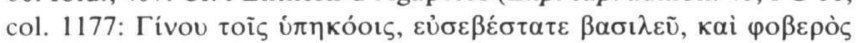

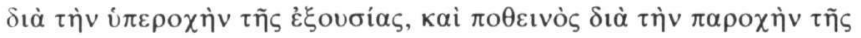

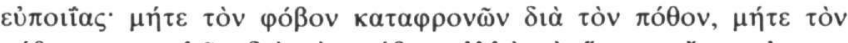

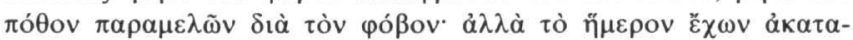

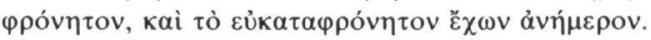

67. Pour le rapport entre pietas et philanthropia/éléèmosynè, cf. le commentaire d'Averil Cameron (n. 15, supra), p. 177-178 (Iust. 2, 399). La philanthropie impériale n'est à son tour qu'une réactivation subordonnée, une mimèsis consciente de la philanthropie divine. Envers le Maître céleste ce n'est qu'une ferme démonstration de piété, l'engrais spirituel du corps investi d'éternité.

68. Iust. 4, 90-205. Cf. le commentaire de Cameron, ibid., p. 197-198. 69. Pour les largesses impériales, cf. Ma c Mullen 1962 (n. 10, supra). 70. Ibid., 215 sq. Pour la métaphore de l'inondation, cf. Dagron 1974 (n. 11, supra), p. 34 n. 2. Cf. les autres allusions à la sparsio, dans le texte de Corippus: Iust. 4, 12 (more nivis sparsurus erat); ibid., 73 (pluat aureus imber).

71. Toutefois, au niveau du parler quotidien, le généreux est toujours désigné en grec par les mots anoichtochérès, haplochérès (cf. skorpo-

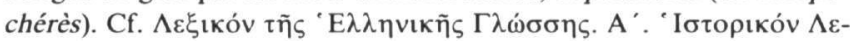

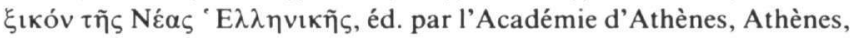

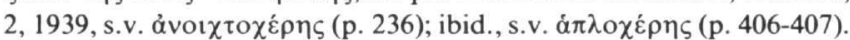
72. Laudatio $S$. Thomaidis 3, Acta Sanctorum, nov. 4, p.

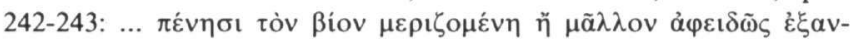

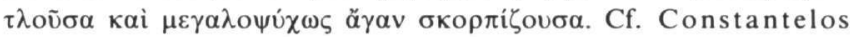
1968 (n. 1, supra), p. 107. La lettre du psaume 111, 9 autorisait l'image littéraire; cependant, ce verset n'est pas illustré dans les psautiers par une image picturale identique (cf. plus loin: Fig. 9).

73. G. Galavaris, The Illustrations of the Homilies of Gregory Nazianzenus, Princeton 1969, p. 63 sq. Cf. PG 35, col. 857 sq. Voir particulièrement la version du Paris. gr. 550 (XIIe s.), fol. 251, où l'on constate l'amplification et la diversification d'une scène qui s'est entre-temps pleinement développée: Galavaris, ibid., fig. 426.

74. Venise, Bibliothèque Marcienne, cod. gr. Z. 540, fol. 5v. Cf. I. Furlan, Codici greci illustrati della Biblioteca Marciana, Milan, 2, 1979 , p. 13 sq.

75. Melbourne, National Gallery of Victoria, cod. Felton 710/5, fol. 5. Cf. Buchthal 1961 (n. 40, supra), p. 5 (la miniature du fol. 5). Pour le codex de Melbourne cf. M. Manion - V. Vines, Medieval and Renaissance Illuminated Manuscripts in Australian Collections, Melbourne - Londres - New York 1984, p. 23-26. Il n'est pas sans intérêt de noter que le geste est ici prêté à un personnage allégorique. Sur la même planche, dans les deux manuscrits, apparaissent d'ailleurs Bienfaisance (Eupoiïa) et Reconnaissance (Eugnômosynè). Signalons enfin que, versant des monnaies, Eléèmosynè ne tient pas de bourse.

76. Dans les manuscrits de Melbourne et de Venise, le collège des vertus fait suite aux douze mois, également personnifiés, —ou, plutôt, rendus par la figuration de diverses occupations périodiques (cf. Buchthal, ibid.; Furlan, ibid.). Un semeur figure le mois de décembre.

77. Cf. Antonopoulos 1981 (n. 2, supra). 


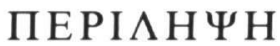

\section{AПO THN LIBERALITAS $\Sigma$ THN EAEHMO $\Sigma$ YNH:

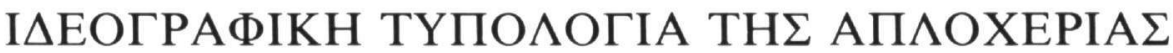

\section{$\Xi$}

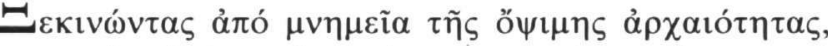

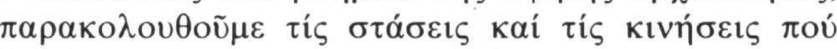

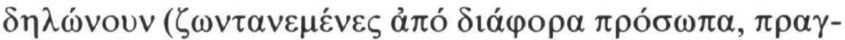

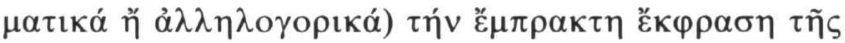

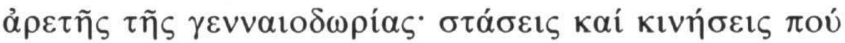

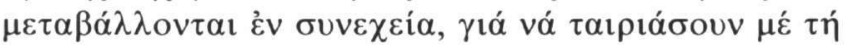

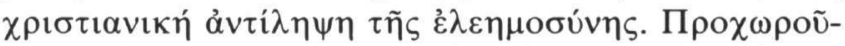

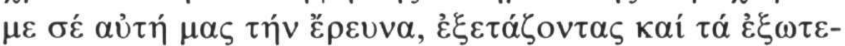

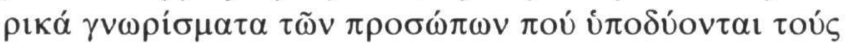

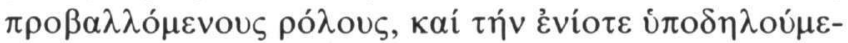

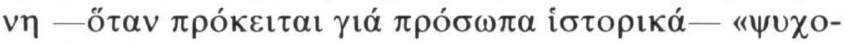

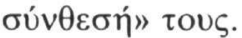

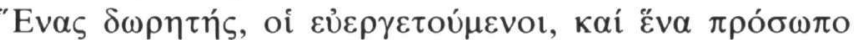

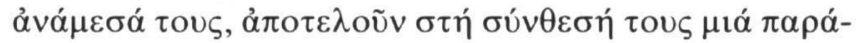

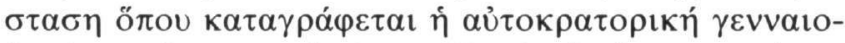

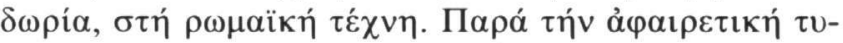

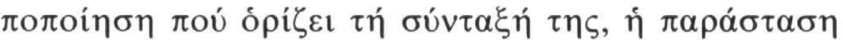

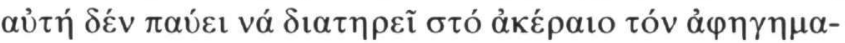

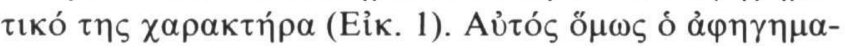

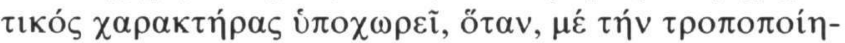

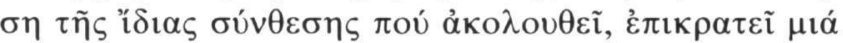

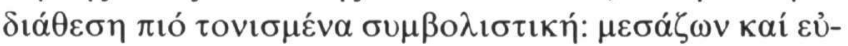

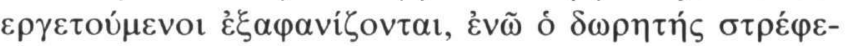

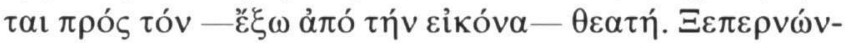

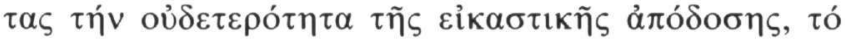

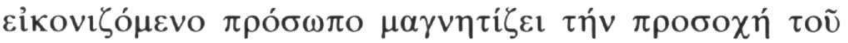

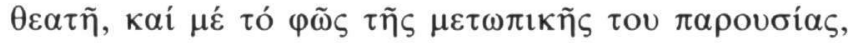

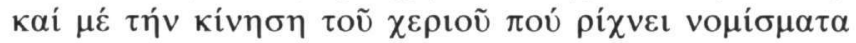

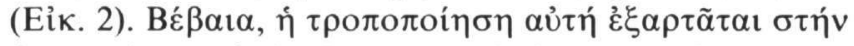

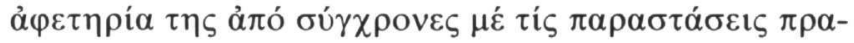

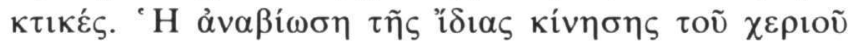

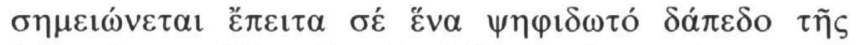

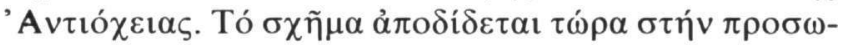

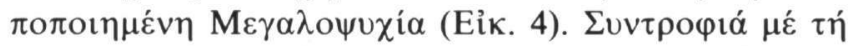

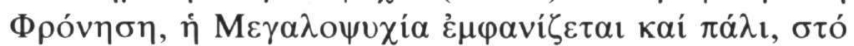

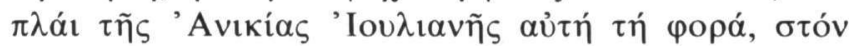

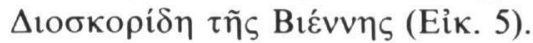

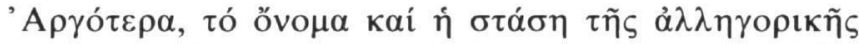

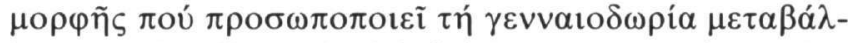

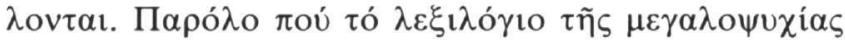

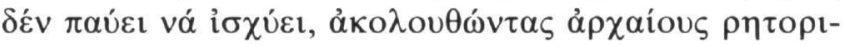

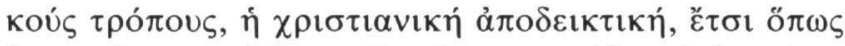

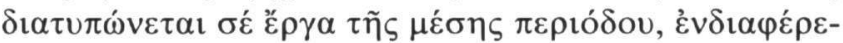

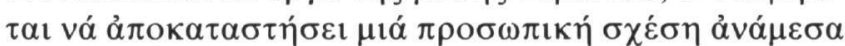

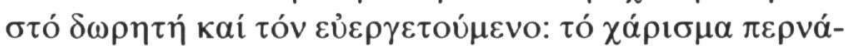

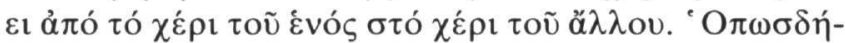

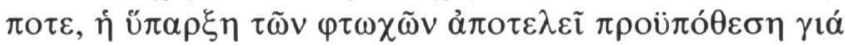

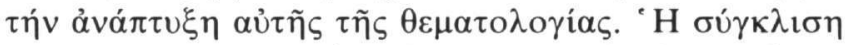

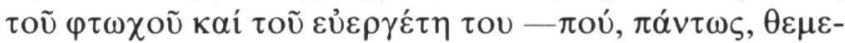

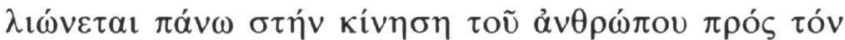

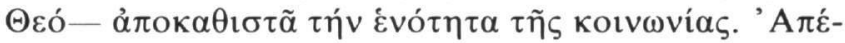

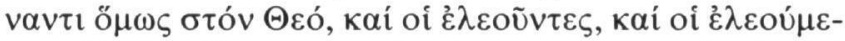

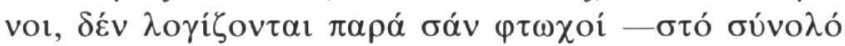

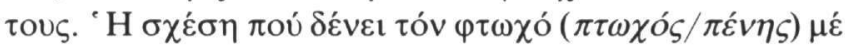

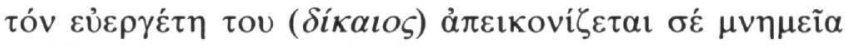

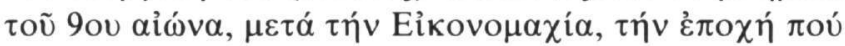

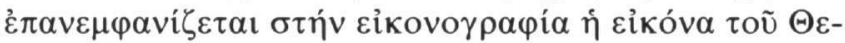

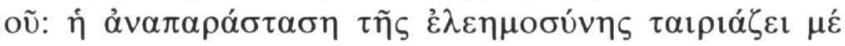

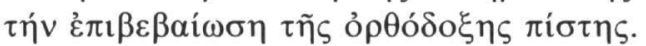

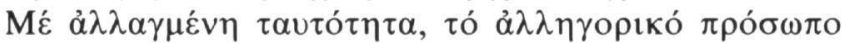

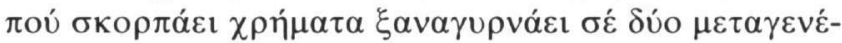

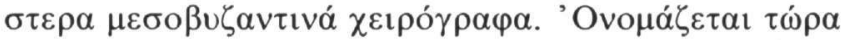

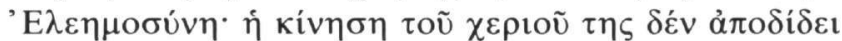

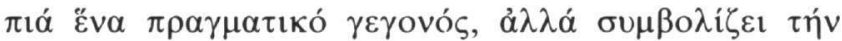

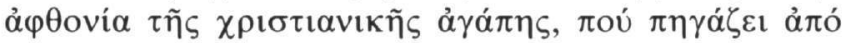

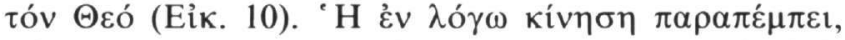

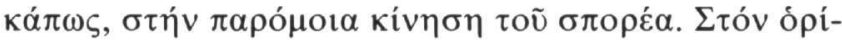

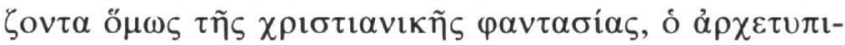

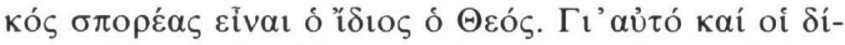

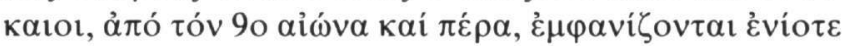

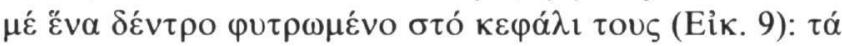

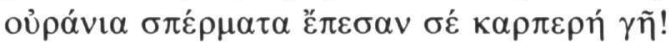

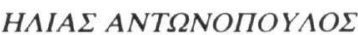

